Portland State University

PDXScholar

\title{
A Class of Discontinuous Petrov-Galerkin Methods. Part III: Adaptivity
}

Leszek Demkowicz

University of Texas at Austin

Jay Gopalakrishnan

Portland State University, gjay@pdx.edu

Antti H. Niemi

University of Texas at Austin

Follow this and additional works at: https://pdxscholar.library.pdx.edu/mth_fac

Part of the Computational Engineering Commons, and the Mathematics Commons

Let us know how access to this document benefits you.

\section{Citation Details}

Published as: Demkowicz, L., Gopalakrishnan, J., \& Niemi, A. H. (2012). A class of discontinuous Petrov-Galerkin methods. Part III: Adaptivity. Applied numerical mathematics, 62(4), 396-427.

This Post-Print is brought to you for free and open access. It has been accepted for inclusion in Mathematics and Statistics Faculty Publications and Presentations by an authorized administrator of PDXScholar. Please contact us if we can make this document more accessible: pdxscholar@pdx.edu. 


\title{
A Class of Discontinuous Petrov-Galerkin Methods. Part III: Adaptivity
}

\author{
Leszek Demkowicz ${ }^{\mathrm{a}, *}$, Jay Gopalakrishnan ${ }^{\mathrm{b}}$, Antti H. Niemi ${ }^{\mathrm{a}}$ \\ ${ }^{a}$ Institute for Computational Engineering and Sciences \\ The University of Texas at Austin, Austin, TX 78712, USA \\ ${ }^{b}$ Department of Mathematics \\ University of Florida, FL 32611, USA
}

\begin{abstract}
We continue our theoretical and numerical study on the Discontinuous Petrov-Galerkin method with optimal test functions in context of $1 \mathrm{D}$ and $2 \mathrm{D}$ convection-dominated diffusion problems and $h p$-adaptivity. With a proper choice of the norm for the test space, we prove robustness (uniform stability with respect to the diffusion parameter) and mesh-independence of the energy norm of the FE error for the 1D problem. With $h p$-adaptivity and a proper scaling of the norms for the test functions, we establish new limits for solving convection-dominated diffusion problems numerically: $\epsilon=10^{-11}$ for $1 \mathrm{D}$ and $\epsilon=10^{-7}$ for $2 \mathrm{D}$ problems. The adaptive process is fully automatic and starts with a mesh consisting of few elements only.
\end{abstract}

Keywords: convection-dominated diffusion, $h p$-adaptivity, Discontinuous Petrov-Galerkin

\section{Introduction}

We begin with a short review of two main concepts behind the Discontinuous Petrov Galerkin (DPG) Method with Optimal Test Functions introduced in [1]: the abstract idea of optimal test functions, and its practical realization within the DPG method. We follow then with a short comparison with stabilized methods and a discussion of the main algorithm. We finish by outlining the scope of this contribution.

\subsection{Petrov-Galerkin Method with Optimal Test Functions}

Variational problem and energy norm. Consider an arbitrary abstract variational problem,

$$
\left\{\begin{array}{l}
u \in U \\
b(u, v)=l(v) \quad \forall v \in V
\end{array}\right.
$$

Here $U, V$ are two real Hilbert spaces and $b(u, v)$ is a continuous bilinear form on $U \times V$,

$$
|b(u, v)| \leq M\|u\|_{U}\|v\|_{V}
$$

that satisfies the inf-sup condition

$$
\inf _{\|u\|_{U}=1} \sup _{\|v\|_{V}=1}|b(u, v)|=: \gamma>0
$$

\footnotetext{
*Corresponding author

Email addresses: leszek@ices.utexas.edu (Leszek Demkowicz), jayg@ufl.edu (Jay Gopalakrishnan), ahniemi@ices.utexas.edu (Antti H. Niemi)
} 
A continuous linear functional $l \in V^{\prime}$ represents the load. If the null space of the adjoint operator,

$$
V_{0}:=\{v \in V: b(u, v)=0 \quad \forall u \in U\}
$$

is non-trivial, the functional $l$ is assumed to satisfy the compatibility condition:

$$
l(v)=0 \quad \forall v \in V_{0}
$$

By the Banach Closed-Range Theorem (see e.g. [2]), problem (1.1) possesses a unique solution $u$ that depends continuously upon the data - functional $l$. More precisely,

$$
\|u\|_{U} \leq \frac{M}{\gamma}\|l\|_{V^{\prime}}
$$

If we define an alternative energy norm equivalent to the original norm on $U$,

$$
\|u\|_{E}:=\sup _{\|v\|=1}|b(u, v)|
$$

both the continuity and the inf-sup constant become equal to one. Recalling that the Riesz operator,

$$
R: V \ni v \rightarrow(v, \cdot) \in V^{\prime}
$$

is an isometry from $V$ into its dual $V^{\prime}$, we may characterize the energy norm in an equivalent way as

$$
\|u\|_{E}=\left\|v_{u}\right\|_{V}
$$

where $v_{u}$ is the solution of the variational problem,

$$
\left\{\begin{array}{l}
v_{u} \in V \\
\left(v_{u}, \delta v\right)_{V}=b(u, \delta v) \quad \forall \delta v \in V
\end{array}\right.
$$

with $(\cdot, \cdot)_{V}$ denoting the inner product in the test space $V$.

Optimal test functions. Let $U_{h p} \subset U$ be a finite-dimensional space with a basis $e_{j}=e_{j, h p}, j=1, \ldots, N=$ $N_{h p}$. For each basis trial function $e_{j}$, we introduce a corresponding optimal test (basis) function $\bar{e}_{j} \in V$ that realizes the supremum,

$$
\left|b\left(e_{j}, \bar{e}_{j}\right)\right|=\sup _{\|v\|_{V}=1}\left|b\left(e_{j}, v\right)\right|
$$

i.e. solves the variational problem,

$$
\left\{\begin{array}{l}
\bar{e}_{j} \in V \\
\left(\bar{e}_{j}, \delta v\right)_{V}=b\left(e_{j}, \delta v\right) \quad \forall \delta v \in V
\end{array}\right.
$$

The test space is now defined as the span of the optimal test functions, $\bar{V}_{h p}:=\operatorname{span}\left\{\bar{e}_{j}, j=1, \ldots, N\right\} \subset V$. The Petrov-Galerkin discretization of the problem (1.1) with the optimal test functions takes the form:

$$
\left\{\begin{array}{l}
u_{h p} \in U_{h p} \\
b\left(u_{h p}, v_{h p}\right)=l\left(v_{h p}\right) \quad \forall v_{h p} \in \bar{V}_{h p}
\end{array}\right.
$$

It follows from the construction of the optimal test functions that the discrete inf-sup constant

$$
\inf _{\left\|u_{h p}\right\|_{E}=1} \sup _{\left\|v_{h p}\right\|=1}\left|b\left(u_{h p}, v_{h p}\right)\right|
$$


is also equal to one. Consequently, Babuška's Theorem [3] implies that

$$
\left\|u-u_{h p}\right\|_{E} \leq \inf _{w_{h p} \in U_{h p}}\left\|u-w_{h p}\right\|_{E}
$$

i.e., the method delivers the best approximation error in the energy norm.

The construction of optimal test functions implies also that the global stiffness matrix is symmetric and positive-definite. Indeed,

$$
b\left(e_{i}, \bar{e}_{j}\right)=\left(\bar{e}_{i}, \bar{e}_{j}\right)_{V}=\left(\bar{e}_{j}, \bar{e}_{i}\right)_{V}=b\left(e_{j}, \bar{e}_{i}\right)
$$

Once the approximate solution has been obtained, the energy norm of the FE error $e_{h p}:=u-u_{h p}$ is determined by solving again the variational problem

$$
\left\{\begin{array}{l}
v_{e_{h p}} \in V \\
\left(v_{e_{h p}}, \delta v\right)_{V}=b\left(u-u_{h p}, \delta v\right)=l(\delta v)-b\left(u_{h p}, \delta v\right) \quad \forall \delta v \in V
\end{array}\right.
$$

We shall call the solution $v_{e_{h p}}$ to the problem (1.17) as the the error representation function since we have

$$
\left\|e_{h p}\right\|_{E}=\left\|v_{e_{h p}}\right\|_{V}
$$

Notice that the energy norm of the error can be computed (approximately, see below) without knowledge of the exact solution. Indeed, the energy norm of the error is nothing else than a properly defined norm of the residual.

Approximate optimal test functions. In practice, the optimal test functions $\bar{e}_{j}$ and the error representation function $v_{e_{h p}}$ are computed approximately using a sufficiently large "enriched" subspace $V_{h p} \subset V$. The optimal test space $\bar{V}_{h p}$ is then a subspace of the enriched test space $V_{h p}$. For instance, if elements of order $p$ are used, the enriched test space may involve polynomials of order $p+\Delta p$. The approximate optimal test space forms a proper subspace of the enriched space and it should not be confused with the enriched space itself, though.

\subsection{Discontinuous Petrov Galerkin Method}

For conforming discretizations, approximate solution of the variational problem (1.12),

$$
\left\{\begin{array}{l}
\bar{e}_{j} \in V_{h p} \\
\left(\bar{e}_{j}, \delta v\right)_{V}=b\left(e_{j}, \delta v\right) \quad \forall \delta v \in V_{h p}
\end{array}\right.
$$

leads to a global system of equations and, consequently, the whole discussed concept has little practical value. The situation changes, if the above methodology is applied in context of the Discontinuous Petrov Galerkin (DPG) method introduced by Bottasso, Micheletti, Sacco and Causin $[4,5,6,7]$. The starting point of the method is a system of first-order differential equations. The equations are multiplied by test functions, integrated over the domain, and then integrated by parts. Contrary to classical variational formulations where some of the equations are relaxed and some others are enforced in a strong form, in the DPG method all equations are considered in a weak sense. Formulations like this are sometimes termed as ultra-weak variational formulations.

The key point of the DPG method of Bottasso et al. is anyway the treatment of the boundary flux terms resulting from integration by parts as independent unknowns. This is in contrast to most of the discontinuous Galerkin formulations found in the literature where the boundary terms are related to the field variables supported over the elements using the concept of numerical flux. The departure from the classical path means that the test and trial functions cannot be chosen within the same spaces so that we are indeed led to a Petrov-Galerkin formulation. The approach is rather transparent since the characteristics of the resulting method are dictated mainly by the choice of the finite element spaces for the test and trial functions without being affected by the specific expressions of the numerical fluxes. In the works of Bottasso and co-workers 
the FE spaces are chosen explicitly so as to obtain methods with desired optimality properties. However, in the first two papers of the present series $[8,1]$, we have taken few steps further by demonstrating how the framework can actually be utilized to compute the optimal test functions once a proper trial space has been chosen. The optimal test functions turn out to be non-polynomials generally, but they can be approximated in a computationally efficient manner by using higher order polynomial spaces element-wise.

Inversion of the Riesz operator in context of the DPG method and discontinuous test functions, is done on the element level by solving problem (1.12) (for the optimal test functions) and problem (1.17)(for the error representation function). In 2D, this involves solving a variational problem set in $\boldsymbol{H}(\operatorname{div}, K) \times H^{1}(K)$. In practice, we can solve those problems only approximately by discretizing space $\boldsymbol{H}(\operatorname{div}, K) \times H^{1}(K)$. The simplest practical solution was to solve the problems using the $p$-method. With element trial shape functions being polynomials of order $p$, we solve for the approximate optimal test functions in the "enriched" space $\mathcal{P}^{p+\Delta p}$. The minimum value for $\Delta p$ is such that the dimension of the enriched space is greater or equal to the dimension of the trial space (for field variables and boundary fluxes combined). Otherwise, the ultimate element stiffness matrix would be singular. Notice that the discretization of the local (selfadjoint and coercive) problems is done using the standard Bubnov-Galerkin method. Standard estimates for the $p$-method indicate that with the $\Delta p \rightarrow \infty$, the approximate optimal test functions (approximate error representation function) will converge to the exact test functions (error representation function). The $p$ enrichment was the easiest approach to code. Alternatively, we could use an element subgrid discretization, i.e. an $h$ - or $h p$-refined element. In principle, we could resolve the optimal test functions even with a controlled accuracy, using adaptive methods. The optimal test functions are thus elements of the enriched space and so is the space of element (approximate) optimal test functions. With the dimension of the enriched space exceeding the dimension of the trial space, the element test space is only a proper subspace of the enriched space.

The square of the global error given by (1.18) is equal to the sum of the corresponding element contributions,

$$
\left\|e_{h p}\right\|_{E}^{2}=\left\|v_{e_{h p}}\right\|_{V}^{2}=\sum_{K} \underbrace{\left\|v_{e_{h p}}\right\|_{V(K)}^{2}}_{e_{K}}
$$

where the element error representation function $v_{e_{h p}}$ is computed by solving a local counterpart of (1.17) using the element enriched space. The individual element contributions $e_{K}$ serve as element error indicators and form the basis for adaptive mesh refinement.

\section{Comparison with DG and other Stabilized Methods}

We refer to $[1,8,9]$ for a partial review of related DG literature. The DG methods are based on the concept of numerical flux of Peter Lax whose definition requires typically stronger regularity assumptions on the solution than implied by the energy setting, see e.g. the original contributions of Cockburn and Shu $[10,11]$. For instance, use of traces for the standard DG method for the convection problem is illegal at the continuous level - the $L^{2}$ functions do not admit traces (see the discussion in [8]). The numerical flux technique can thus be viewed as means of stabilization coming from the boundary of the element (typical DG methods use Bubnov-Galerkin discretization) and in this sense the DG methods fall into a wider class of stabilized FE methods. The breakthrough concept of stabilized FE methods which started with the Streamline-Diffusion Petrov Galerkin (SUPG) method of Hughes and Brooks [12], has been at the forefront of FE research in CFD for the last three decades. In particular, in the case of "bubble methods", stabilization comes from introducing and condensing out additional bubble shape functions (i.e. functions vanishing on the element boundary) $[13,14]$. You might say, that the stabilization comes from the element interior. Remarkably, the bubble techniques may lead to the same discrete equations as the SUPG method [15]. Except for DPG methods (see [16, 17, 18], majority of stabilized methods is restricted to low order elements. The common property of all stabilized methods is a proper modification of the bilinear and linear form at the discrete level without violating the consistency. Contrary to those methods, the stability of the DPG method with optimal test functions comes only from the optimal testing (computed on the fly). No modifications are made to the original bilinear and linear forms. The DPG method is directly linked with 
the concept of generalized least squares method see e.g. [19]. If we write the original variational problem in the operator form,

$$
B u=l, \quad B: U \rightarrow V^{\prime}, \quad<B u, v>=b(u, v)
$$

precondition it with the inverse of the Riesz operator in test space $V$,

$$
R_{V}^{-1} B u=R_{V}^{-1} l, \quad R_{V}^{-1} B: U \rightarrow V
$$

and apply the least squares in the test space $V$,

$$
\left\|R_{V}^{-1} B u_{h p}-R_{V}^{-1} l\right\|_{V}^{2} \rightarrow \min
$$

we obtain exactly the DPG method.

Implementation of the DPG method. Implementation of DPG with optimal testing requires minimal changes when compared with standard hybrid methods. All numerical experiments presented in this paper were done with a standard frontal solver that requires returning for each element local-to-global connectivities for element degrees-of-freedom (d.o.f.), and the corresponding element matrices. Similarly to Raviart-Thomas elements, the DPG method involves local (interior) d.o.f. related to "field variables" and d.o.f. corresponding to the DPG fluxes that live on element edges and are shared by adjacent elements. The corresponding trial shape functions are only globally $L^{2}$-conforming, so no global continuity need to be enforced. The essential difference comes in the element routine. Using the enriched space polynomials, we set up the auxiliary variational problem (1.12) and solve it approximately using the standard Bubnov-Galerkin method and the enriched space. One can think of a miniature spectral FE problem defined on one element. Note the following simple observations.

- The local problem (1.12) involves multiple right-hand sides. We perform the $L U$ decomposition of the stiffness matrix once and follow with multiple back substitutions.

- Once the optimal shape functions have been determined, the actual problem element stiffness matrix is computed utilizing relation (1.16). We integrate for the load vector, however, using the standard procedure.

- In the case of uniform meshes, both in terms of geometry, material properties and polynomial order, the element optimal test functions can be precomputed.

Computation of error representation function and the corresponding element contribution to the energy error is done using the same algorithm except for a different load to the local problem, comp. (1.17). Notice a similarity with the implicit a-posteriori error estimation techniques using equilibriated residuals [20]. The main difference lies in the fact that, in the case of the DPG method, there is no need to equlibriate the element residuals.

Scope of this paper. The present work is a continuation of [1] and focuses on convection-dominated diffusion problems. In Section 2, we present a refined stability analysis for the 1D problem and show how a proper choice of the norm for the test functions leads to a robust method, i.e. a method uniformly stable with respect to the diffusion parameter $\epsilon$. We also prove a fundamental property of the error representation function global continuity. The main focus of the paper though is automatic adaptivity. Section 3 presents numerical results for the 1D problem, and in Section 4 we share our 2D experience. With a clever choice of norms for the test functions we achieve not only the robustness but have also been able to push the existing limits for solving convection-dominated diffusion problems using high-order finite elements with double precision arithmetic. Concerning the lowest possible value of the diffusion parameter, the new "records" are $\epsilon=10^{-11}$ for $1 \mathrm{D}$, and $\epsilon=10^{-7}$ for $2 \mathrm{D}$ problems. Most importantly, the whole mesh optimization process is completely automatic and starts with an initial mesh consisting of few elements only. 


\section{Convection-Dominated Diffusion in 1D}

In this section, we continue our study on a $1 \mathrm{D}$ convection-dominated diffusion model problem initiated in [1]. We demonstrate that, with a proper choice of the norm for the test functions, we may obtain a robust DPG method, i.e. a method whose stability properties are uniform in the diffusion parameter $\epsilon$. More precisely, we show that the standard $L^{2}$-norm of the velocity $u$ and the stress $\sigma=\epsilon u^{\prime}$ is bounded by the DPG energy norm times an $\epsilon$-independent constant of order one.

In general, the expression of the DPG energy norm is mesh dependent. If we $h$-refine the mesh, the expression changes and there is no guarantee that the error in this norm would decrease. However, if we work with the standard (possibly weighted) $H^{1}$-norm for the test functions,

$$
\|\boldsymbol{v}\|_{K}^{2}=\int_{K}\left(\left|\boldsymbol{v}^{\prime}\right|^{2}+|\boldsymbol{v}|^{2}\right) \alpha(x) d x
$$

the corresponding energy norm of the FE error turns out to be mesh-independent in the following sense: We will show that the DPG energy norm of the FE error for an arbitrary $h p$ mesh coincides with the spectral (one element) DPG energy norm of the error.

$1 D$ model problem. Given a right-hand side $f(x), x \in(0,1)$ and inflow data $u_{0}$, we consider the problem:

$$
\begin{cases}u(0)=u_{0}, & u(1)=0 \\ \frac{1}{\epsilon} \sigma-u^{\prime} & =0 \\ -\sigma^{\prime}+u^{\prime} & =f\end{cases}
$$

Introducing an arbitrary partition,

$$
0=x_{0}<x_{1}<\ldots<x_{k-1}<x_{k}<\ldots<x_{N}=1
$$

we formulate the DPG method as follows. The unknowns include field variables $\sigma_{k}$, $u_{k}$ defined over element $\left(x_{k-1}, x_{k}\right)$ and approximated with (equal) order polynomials $\mathcal{P}^{p}\left(x_{k-1}, x_{k}\right)$ and fluxes $\hat{\sigma}\left(x_{k}\right), \hat{u}\left(x_{k}\right), k=$ $0, \ldots, N$. Fluxes $\hat{u}(0)=u_{0}, \hat{u}(1)=0$ are known from the boundary conditions. For each element $K=$ $\left(x_{k-1}, x_{k}\right)$, we have test functions $(\tau, v)=\left(\tau_{k}, v_{k}\right)$. Polynomial order $p$ may vary from element to element. Consistently with the $L^{2}$-energy setting for $\sigma, u$, the discretization is globally discontinuous.

For each $k=1, \ldots, N$, we satisfy the following variational equations,

$$
\left\{\begin{array}{lcl}
\frac{1}{\epsilon} \int_{x_{k-1}}^{x_{k}} \sigma_{k} \tau & +\int_{x_{k-1}}^{x_{k}} u_{k} \tau^{\prime}-\left.(\hat{u} \tau)\right|_{x_{k-1}} ^{x_{k}}=0 \\
\int_{x_{k-1}}^{x_{k}} \sigma_{k} v^{\prime}-\left.(\hat{\sigma} v)\right|_{x_{k-1}} ^{x_{k}}-\int_{x_{k-1}}^{x_{k}} u_{k} v^{\prime}+\left.(\hat{u} v)\right|_{x_{k-1}} ^{x_{k}}=\int_{x_{k-1}}^{x_{k}} f v
\end{array}\right.
$$

for every optimal test function $\tau, v$. Again, for $k=1, \hat{u}(0)=u_{0}$ is known and is moved to the right-hand side. Similarly, $\hat{u}(1)=0$ in the last equation for $k=N$.

Norm for the test functions. The norm for the test functions is defined element-wise:

$$
\|(\boldsymbol{\tau}, \boldsymbol{v})\|=\left(\sum_{k=1}^{N}\left\|\tau_{k}\right\|_{k}^{2}+\left\|v_{k}\right\|_{k}^{2}\right)^{\frac{1}{2}}
$$

We will work with two choices for the element norms, 
- A weighted $H^{1}$-norm,

$$
\begin{aligned}
\left\|\tau_{k}\right\|_{k}^{2} & =\int_{x_{k-1}}^{x_{k}} \alpha\left(\left|\tau_{k}^{\prime}\right|^{2}+\left|\tau_{k}\right|^{2}\right) d x \\
\left\|v_{k}\right\|_{k}^{2} & \left.=\left.\int_{x_{k-1}}^{x_{k}} \alpha\left(\left|v_{k}^{\prime}\right|^{2}+\mid v_{k}\right)\right|^{2}\right) d x
\end{aligned}
$$

- A mesh-dependent norm,

$$
\begin{aligned}
\left\|\tau_{k}\right\|_{k}^{2} & =\int_{x_{k-1}}^{x_{k}} \alpha\left|\tau_{k}^{\prime}\right|^{2}+\beta_{k}\left|\tau_{k}\left(x_{k}\right)\right|^{2} d x \\
\left\|v_{k}\right\|_{k}^{2} & =\int_{x_{k-1}}^{x_{k}} \alpha\left|v_{k}^{\prime}\right|^{2}+\beta_{k}\left|v_{k}\left(x_{k}\right)\right|^{2} d x
\end{aligned}
$$

Notice that, for a globally continuous function, the norm corresponding to element norm (2.29) reduces to a mesh-independent global (spectral) norm, whereas in the second case, it does not. Weights $\alpha=\alpha(x), \beta_{k}, k=$ $1, \ldots, N$ are to be selected.

\section{Global Continuity of the Error Representation Function}

We start with a notation. Let $\mathcal{U}=(\boldsymbol{\sigma}, \boldsymbol{u}, \hat{\boldsymbol{\sigma}}, \hat{\boldsymbol{u}})$ denote the exact solution,

$$
\begin{aligned}
\boldsymbol{\sigma} & :=\left(\sigma_{1}, \ldots, \sigma_{N}\right) \\
\boldsymbol{u} & :=\left(u_{1}, \ldots, u_{N}\right) \\
\hat{\boldsymbol{\sigma}} & :=\left(\hat{\sigma}\left(x_{0}\right), \ldots, \hat{\sigma}\left(x_{N}\right)\right) \\
\hat{\boldsymbol{u}} & :=\left(\hat{u}\left(x_{1}\right), \ldots, \hat{u}\left(x_{N-1}\right)\right)
\end{aligned}
$$

and $\mathcal{U}_{h p}$ the corresponding approximate counterpart. Consider two neighboring elements $\left(x_{k-1}, x_{k}\right),\left(x_{k}, x_{k+1}\right)$. Let $\left(\tau_{\hat{\sigma}_{k}}, v_{\hat{\sigma}_{k}}\right)$ be the optimal, vector-valued test function corresponding to flux $\hat{\sigma}_{k}$, spanned over the two elements. We have the standard orthogonality condition for the error function $\mathcal{E}_{h p}:=\mathcal{U}-\mathcal{U}_{h p}$,

$$
b\left(\mathcal{U}-\mathcal{U}_{h p},\left(\tau_{\hat{\sigma}_{k}}, v_{\hat{\sigma}_{k}}\right)\right)=b_{k}\left(\mathcal{U}-\mathcal{U}_{h p},\left(\tau_{\hat{\sigma}_{k}}, v_{\hat{\sigma}_{k}}\right)\right)+b_{k+1}\left(\mathcal{U}-\mathcal{U}_{h p},\left(\tau_{\hat{\sigma}_{k}}, v_{\hat{\sigma}_{k}}\right)\right)=0
$$

Here $b_{k}$ denotes the $k$-th element contribution to the bilinear form defined by $(2.27)$. Let $\left(\phi_{k}, \psi_{k}\right)$ be now the error representation function for the $k$-th element,

$$
\left\{\begin{array}{l}
\left(\phi_{k}, \psi_{k}\right) \in V\left(\left(x_{k-1}, x_{k}\right)\right) \\
\left(\left(\phi_{k}, \psi_{k}\right),(\delta \phi, \delta \psi)\right)_{k}=b_{k}\left(\mathcal{E}_{h p},(\delta \phi, \delta \psi)\right), \quad \forall(\delta \phi, \delta \psi) \in V\left(\left(x_{k-1}, x_{k}\right)\right)
\end{array}\right.
$$

Here $(\cdot, \cdot)_{k}$ denotes the inner product corresponding to element norm $\|\cdot\|_{k}$, and $V\left(\left(x_{k-1}, x_{k}\right)\right)$ is the element test space. For the exact optimal test functions $V\left(\left(x_{k-1}, x_{k}\right)\right)=H^{1}\left(\left(x_{k-1}, x_{k}\right)\right)$, for the approximate test functions, $V\left(\left(x_{k-1}, x_{k}\right)\right)=\mathcal{P}^{p+\Delta p}\left(\left(x_{k-1}, x_{k}\right)\right)$ where $p$ is the element order of approximation that varies with element, and $\Delta p$ is a global increment in $p$ defining the enriched space. We have,

$$
\left(\left(\phi_{k}, \psi_{k}\right),\left(\tau_{\hat{\sigma}_{k}}, v_{\hat{\sigma}_{k}}\right)\right)_{k}+\left(\left(\phi_{k+1}, \psi_{k+1}\right),\left(\tau_{\hat{\sigma}_{k}}, v_{\hat{\sigma}_{k}}\right)\right)_{k+1}=b\left(\mathcal{U}-\mathcal{U}_{h p},\left(\tau_{\hat{\sigma}_{k}}, v_{\hat{\sigma}_{k}}\right)\right)=0
$$

On the other side, the definition of optimal test functions implies that

$$
\left(\left(\tau_{\hat{\sigma}_{k}}, v_{\hat{\sigma}_{k}}\right),(\delta \phi, \delta \psi)\right)_{k}=-\delta \psi\left(x_{k}\right), \quad \forall(\delta \phi, \delta \psi) \in V\left(\left(x_{k-1}, x_{k}\right)\right)
$$

and

$$
\left(\left(\tau_{\hat{\sigma}_{k}}, v_{\hat{\sigma}_{k}}\right),(\delta \phi, \delta \psi)\right)_{k+1}=\delta \psi\left(x_{k}\right), \quad \forall(\delta \phi, \delta \psi) \in V\left(\left(x_{k}, x_{k+1}\right)\right)
$$

Selecting the error representation functions $\left(\phi_{k}, \psi_{k}\right)$ and $\left(\phi_{k+1}, \psi_{k+1}\right)$ for $(\delta \phi, \delta \psi)$ above, and summing up the two equations, we get

$$
-\psi_{k}\left(x_{k}\right)+\psi_{k+1}\left(x_{k}\right)=0
$$


In a similar way, we use the optimal test function corresponding to flux $\hat{u}\left(x_{k}\right)$ to demonstrate that $\phi_{k}\left(x_{k}\right)-$ $\phi_{k+1}\left(x_{k}\right)=0$ as well. Application of the same argument to the fluxes at the endpoints $x_{0}=0$ and $x_{N}=1$ shows that the corresponding error representation function vanishes there. The discussed result is very general and it holds for any choice of inner products for the test functions and other systems of differential equations as well.

Continuity of the error representation function has an important implication. Solution of the global problem

$$
\left\{\begin{array}{l}
(\phi, \psi) \in V((0,1)) \\
((\phi, \psi),(\delta \phi, \delta \psi))=b\left(\mathcal{E}_{h p},(\delta \phi, \delta \psi)\right), \quad \forall(\delta \phi, \delta \psi) \in V((0,1))
\end{array}\right.
$$

leads to a error representation function $(\phi, \psi)$ where $\phi, \psi$ coincide with the unions of the element error representation functions $\phi_{k}, \psi_{k}$. In order to see it, consider globally continuous test functions $(\delta \tau, \delta v)$ and sum up the local problems corresponding to element error representation functions $\left(\tau_{k}, v_{k}\right)$ to obtain

$$
\begin{aligned}
(\tau, \delta \tau)=\sum_{k=1}^{N}\left(\tau_{k}, \delta \tau\right)_{k} & =\sum_{k=1}^{N}\left\{\frac{1}{\epsilon} \int_{x_{k-1}}^{x_{k}} \sigma_{k} \delta \tau+\int_{x_{k-1}}^{x_{k}} u_{k} \delta \tau^{\prime}-\left.[\hat{u} \delta \tau]\right|_{x_{k-1}} ^{x_{k}}\right\} \\
& =\frac{1}{\epsilon} \int_{0}^{1} \sigma \delta \tau+\int_{0}^{1} u \delta \tau^{\prime} \\
(v, \delta v)=\sum_{k=1}^{N}\left(v_{k}, \delta v\right)_{k} & =\sum_{k=1}^{N}\left\{\int_{x_{k-1}}^{x_{k}} \sigma_{k} \delta v^{\prime}-\left.[\hat{\sigma} \delta v]\right|_{x_{k-1}} ^{x_{k}}-\int_{x_{k-1}}^{x_{k}} u_{k} \delta v^{\prime}-\left.[\hat{u} \delta v]\right|_{x_{k-1}} ^{x_{k}}\right\} \\
& =\int_{0}^{1} \sigma \delta v^{\prime}-\int_{0}^{1} u \delta v^{\prime}
\end{aligned}
$$

where $\sigma$ and $u$ represent unions of $\sigma_{k}$ and $u_{k}$, respectively. In other words, continuous test functions $\delta \tau, \delta v$ "do not see" the jump terms corresponding to inter-element fluxes. This has an important consequence in case of (2.29) because then, for continuous functions, the global norm (2.28) obtained by summing up the element norms coincides with the spectral, one-element norm. Consequently, the DPG energy norm of the FE error also coincides with the spectral energy norm. In general, convergence analysis of discontinuous Galerkin methods is based on mesh-dependent norms and, usually, one has to work rather hard to bound those from below by standard $L^{2}$-type norms. For the DPG method with optimal test functions, at least in the $1 \mathrm{D}$ case, the analysis reduces to the spectral case only.

Another important consequence is the behavior of the energy norm of the error during refinements. As the DPG method with optimal test functions delivers the best approximation in the energy norm, the error cannot increase for a $p$-refined mesh. The discussed result implies that this holds true for $h$-refinements as well. Continuity of the error representation function and non-increase of the error are, of course, true only under the assumption of exact integration and arithmetic and, for this reason, the lack of either in practical computation is a great indicator of round off error effects.

\section{Spectral Stability Analysis}

We begin with a slight generalization of the stability analysis for the spectral case presented in [1] and norm (2.30) with $\beta=1$. In particular, we will find a suitable choice for weight $\alpha$ to guarantee the robustness of the method.

Following the reasoning in [1] (see also Appendix A), we derive the explicit formula for the energy norm,

$$
\begin{gathered}
\|(\sigma, u, \hat{\sigma}(0), \hat{\sigma}(1))\|_{E}^{2}= \\
\left\|\frac{1}{\epsilon} \int_{0}^{x} \sigma(s) d s-u(x)\right\|_{1 / \alpha}^{2}+\|-\sigma(x)+u(x)+\hat{\sigma}(0)\|_{1 / \alpha}^{2}+\left|\frac{1}{\epsilon} \int_{0}^{1} \sigma(s) d s\right|^{2}+|-\hat{\sigma}(1)+\hat{\sigma}(0)|^{2}
\end{gathered}
$$

where $\|\cdot\|_{1 / \alpha}$ denotes the weighted $L^{2}$-norm with weight $1 / \alpha$. The use of norm (2.30) is essential in establishing the explicit formula for the energy norm. 
Formula (2.40) and triangle inequality lead to:

$$
\begin{aligned}
\left\|\frac{1}{\epsilon} \int_{0}^{x} \sigma-\sigma(x)+\hat{\sigma}(0)\right\|_{1 / \alpha}^{2} & \leq\|(\sigma, u, \hat{\sigma}(0), \hat{\sigma}(1))\|_{E}^{2} \\
\left|\frac{1}{\epsilon} \int_{0}^{1} \sigma\right|^{2} & \leq\|(\sigma, u, \hat{\sigma}(0), \hat{\sigma}(1))\|_{E}^{2}
\end{aligned}
$$

Denoting,

we solve for $\sigma$ to obtain,

$$
\frac{1}{\epsilon} \int_{0}^{x} \sigma-\sigma(x)+\hat{\sigma}(0)=: g(x), \quad \frac{1}{\epsilon} \int_{0}^{1} \sigma=: A
$$

$$
\sigma(x)=\hat{\sigma}(0) e^{\frac{x}{\epsilon}}-g(x)-\frac{1}{\epsilon} \int_{0}^{x} e^{\frac{x-s}{\epsilon}} g(s) d s
$$

Integrating both sides over $(0,1)$ and dividing by $\epsilon$, we get

$$
A=\hat{\sigma}(0)\left(e^{\frac{1}{\epsilon}}-1\right)-\frac{1}{\epsilon} \int_{0}^{1} e^{\frac{1-s}{\epsilon}} g(s) d s
$$

Solving for $\hat{\sigma}(0)$, we obtain,

$$
\hat{\sigma}(0)=A \frac{e^{-\frac{1}{\epsilon}}}{1-e^{-\frac{1}{\epsilon}}}+\frac{1}{\epsilon\left(1-e^{-\frac{1}{\epsilon}}\right)} \int_{0}^{1} e^{-\frac{s}{\epsilon}} g(s) d s
$$

Now comes the main point. If we control function $g$ only in the $L^{2}$-norm, then the stability constant reflecting dependence $\hat{\sigma}(0)$ upon $g$ is of order $\epsilon^{-\frac{1}{2}}$, i.e. the stability is not uniform with respect to $\epsilon$ (the method is not robust). If, however, function $g$ is controlled in a weighted norm, we have a chance for robustness. Indeed, choosing for instance

$$
\alpha(x)=\left\{\begin{array}{cc}
\frac{\epsilon}{2} & 0<s<-\frac{\epsilon}{2} \ln \left(\frac{\epsilon}{2}\right) \\
1 & -\frac{\epsilon}{2} \ln \left(\frac{\epsilon}{2}\right)<s<1
\end{array}\right.
$$

we have (see Appendix B),

$$
\left|\int_{0}^{1} e^{-\frac{s}{\epsilon}} g(s) d s\right| \leq \sqrt{2} \epsilon\|g\|_{1 / \alpha}
$$

which leads to the final estimate,

$$
|\hat{\sigma}(0)| \leq \frac{e^{-\frac{1}{\epsilon}}}{1-e^{-\frac{1}{\epsilon}}} A+\frac{1}{\left(1-e^{-\frac{1}{\epsilon}}\right)}\|g\|_{1 / \alpha}
$$

where $\|\cdot\|_{1 / \alpha}$ is the weighted $L^{2}$-norm with weight $1 / \alpha$.

$L^{2}$-stability result for $\sigma$. Substituting expression for $\hat{\sigma}(0)$ into (2.42), we get the final formula for the stress,

$$
\begin{aligned}
\sigma(x) & =\frac{A}{1-e^{-\frac{1}{\epsilon}}} e^{\frac{x-1}{\epsilon}}+\frac{1}{\epsilon\left(1-e^{-\frac{1}{\epsilon}}\right)} \int_{0}^{1} e^{\frac{x-s}{\epsilon}} g(s) d s-g(x)-\frac{1}{\epsilon} \int_{0}^{x} e^{\frac{x-s}{\epsilon}} g(s) d s \\
& =\frac{A}{1-e^{-\frac{1}{\epsilon}}} e^{\frac{x-1}{\epsilon}}+\frac{e^{-\frac{1}{\epsilon}}}{\epsilon\left(1-e^{-\frac{1}{\epsilon}}\right)} \int_{0}^{x} e^{\frac{x-s}{\epsilon}} g(s) d s-g(x)+\frac{1}{\epsilon\left(1-e^{-\frac{1}{\epsilon}}\right)} \int_{x}^{1} e^{\frac{x-s}{\epsilon}} g(s) d s
\end{aligned}
$$

The $L^{2}$-norm of $\sigma$ is bounded in terms of the constant $A$ and the $L^{2}$-norm of $g$. We have shown in [1] that the corresponding constants are independent of $\epsilon$, i.e. the method is robust in $\sigma$ without using the weights. Since the standard $L^{2}$-norm of $g$ is controlled by the weighted norm $\|\cdot\|_{1 / \alpha}$, we also can claim corresponding estimate using the weighted norm for $g$,

$$
\|\sigma\|_{L^{2}} \leq C\left(|A|+\|g\|_{1 / \alpha}\right)
$$

where the constant $C$ is independent of $\epsilon$. 
$L^{2}$-stability result for $u$. Once we can control $\hat{\sigma}(0)$ and $\sigma$ in $L^{2}$-norm, formula for the energy norm implies that we also control $u$ in $L^{2}$-norm. Indeed,

$$
\|-\sigma+u+\hat{\sigma}(0)\|^{2} \leq\|-\sigma+u+\hat{\sigma}(0)\|_{1 / \alpha}^{2} \leq\|(\sigma, u, \hat{\sigma}(0), \hat{\sigma}(1))\|_{E}^{2}
$$

and the triangle inequality imply that

$$
\|u\|^{2} \leq 2\left(\|\sigma\|^{2}+|\hat{\sigma}(0)|^{2}+\|-\sigma+u+\hat{\sigma}(0)\|^{2}\right) \leq C\|(\sigma, u, \hat{\sigma}(0), \hat{\sigma}(1))\|_{E}^{2}
$$

where constant $C$ is independent of $\epsilon$.

\section{FE Stability Result}

Equivalence of the norms (2.29) and (2.30) on the spectral level implies that the proved spectral stability results hold also for the norm (2.29). The global continuity of the error representation function associated with this norm implies that the discussed stability relations between the $L^{2}$ and energy norms are valid on an arbitrary mesh for the FE error function $\mathcal{E}_{h p}=\mathcal{U}-\mathcal{U}_{h p}$.

It is possible to establish a slightly stronger algebraic result by using the norm (2.30) for the test functions. With an appropriate choice of the parameters $\beta_{k}$, the $L^{2}$ norms of both $\sigma$ and $u$ can be bounded by the energy norm of $\mathcal{U}$ times an $\epsilon$-independent constant for any function $\mathcal{U}$ for which the energy is finite. The proof involves the use of "telescoping sums" and can be found in Appendix A.

\section{1D Numerical Experiments}

In this section we present numerical results for the 1D convection-dominated diffusion model problem studied in the previous section. All experiments are reported for the data $f=0$ and $u_{0}=1$. The corresponding solution develops a boundary layer at $x=1$ :

$$
\sigma(x)=-\frac{1}{1-e^{-\frac{1}{\epsilon}}} e^{\frac{x-1}{\epsilon}}, \quad u(x)=\frac{1}{1-e^{-\frac{1}{\epsilon}}}\left(1-e^{\frac{x-1}{\epsilon}}\right)
$$

All reported numerical experiments start with a mesh of four elements of order $p=0$ (piecewise constants). We have used the norm (2.29) for computing the optimal test functions and the error representation functions. In place of the weight $\alpha(x)$ used in the proof of robustness, we have used a simpler choice,

$$
\alpha(x)= \begin{cases}0.1 & x \in\left(0, \frac{1}{4}\right) \\ 1.0 & x \in\left(\frac{1}{4}, 1\right)\end{cases}
$$

The increment $\Delta p$ used to define the enriched space was set to $\Delta p=4$, and the maximum order was set to $p_{\max }=4$. These choices are related to an attempt to determine the minimum diffusion parameter $\epsilon$ for which we can solve the problem numerically using double precision arithmetic.

We have used a so called "poor man greedy $h p$ algorithm" summarized below to guide the mesh refinements. 


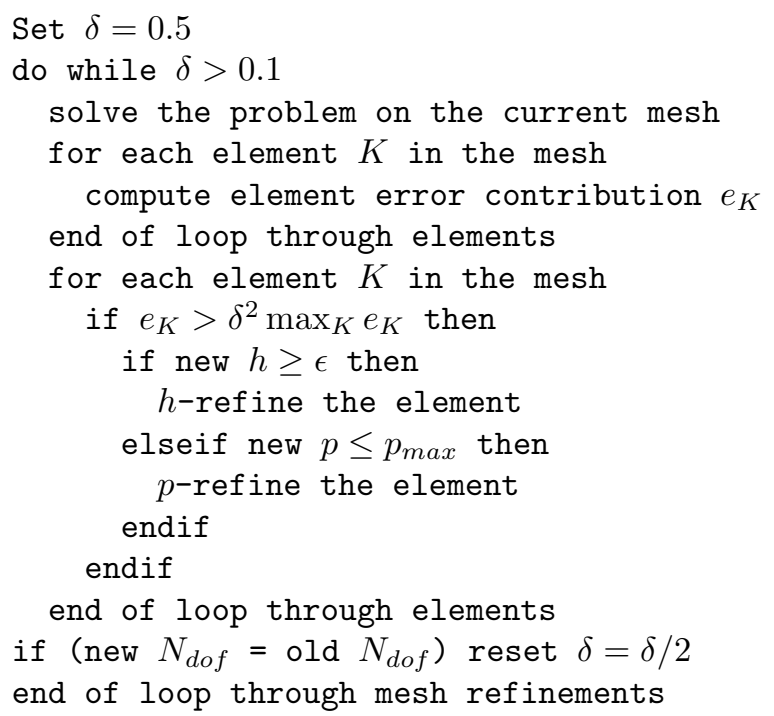

The strategy reflects our old experiments with boundary layers [21], and the rigorous approximability results of Schwab and Suri [22] and Melenk [23] on optimal $h p$ discretizations of boundary layers: we proceed with $h$-refinements until the diffusion scale $\epsilon$ is reached and then continue with $p$-refinements.

We start with an easy case of $\epsilon=10^{-3}$. Fig. 1 presents the corresponding convergence history on a log-log scale, the energy norm of the error vs. the number of degrees-of-freedom (d.o.f.). As predicted by the theory, the error goes monotonically down. The curve displays three different regions. The first one corresponds to a series of $h$-refinements "localizing" the boundary layer and is characterized with relatively slow convergence. The second region, characterized with a rapid convergence, corresponds to the $p$-refinements in the boundary layer and illustrates the power of $p$-refinements on a properly refined mesh. Finally, the last part corresponds to the last few refinements when the mesh restrictions in terms of both minimum element size and maximum polynomial order, become active. Fig. 2 presents a $10 \times$ zoom on the boundary layer revealing the "optimal" $h p$ mesh obtained using our algorithm and perfect resolution of the boundary layer (eye ball norm). Finally, Fig. 3 displays the corresponding error representation function $\phi$. The function is continuous consistently with the theory.

We continue now with a more challenging case with $\epsilon=10^{-6}$. Fig. 4 presents the convergence history showing that the error goes monotonically down until the last few refinements where it begins to oscillate. Fig. 5 presents a $10^{5} \times$ zoom on the boundary layer revealing the optimal $h p$ mesh and (still) a perfect resolution of the boundary layer. So far, everything looks OK. If we look however at Fig. 6 showing the error representation function $\phi$, we see that, contrary to the theory, the function is no longer globally continuous. For $\epsilon=10^{-7}$ the method falls apart and the code reports zero pivots in solving local problems for optimal test functions. The loss of continuity of the error representation functions (the same behavior is observed for function $\psi$ ) is visible, starting already with $\epsilon=10^{-4}$. For elements resolving the boundary layer, element size $h=\epsilon$. The ratio of the stiffness and mass terms in formula (2.29) is of order $p^{2} / h^{2}$ and, for $h=10^{-3}, p=4$, is of order $10^{7}$, which corresponds to half of the 14-15 digits available in double precision arithmetic. With $h=10^{-4}$, the overlap is minimized to 5 digits and we begin to observe the effects of round off errors ${ }^{1}$. Apparently, the problems are not limited only to the solution of the element, local problems for the optimal test functions. To investigate the issue, we have employed for local shape functions (a basis for the enriched space used to determine optimal test functions and solve for the error representation functions)

\footnotetext{
${ }^{1}$ The situation resembles experience with penalty methods.
} 


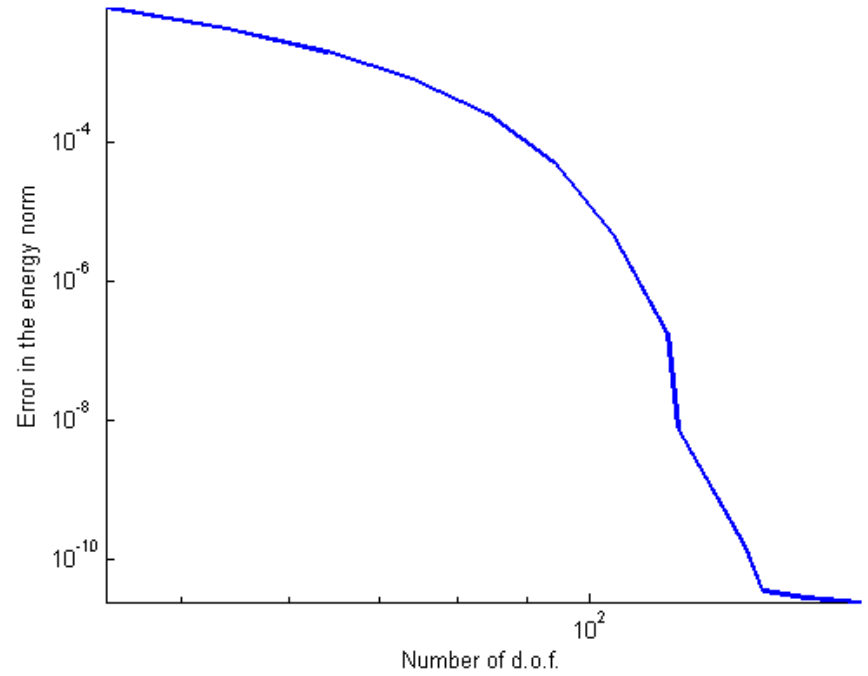

Figure 1: Diffusion parameter $\epsilon=10^{-3}$ : convergence history.

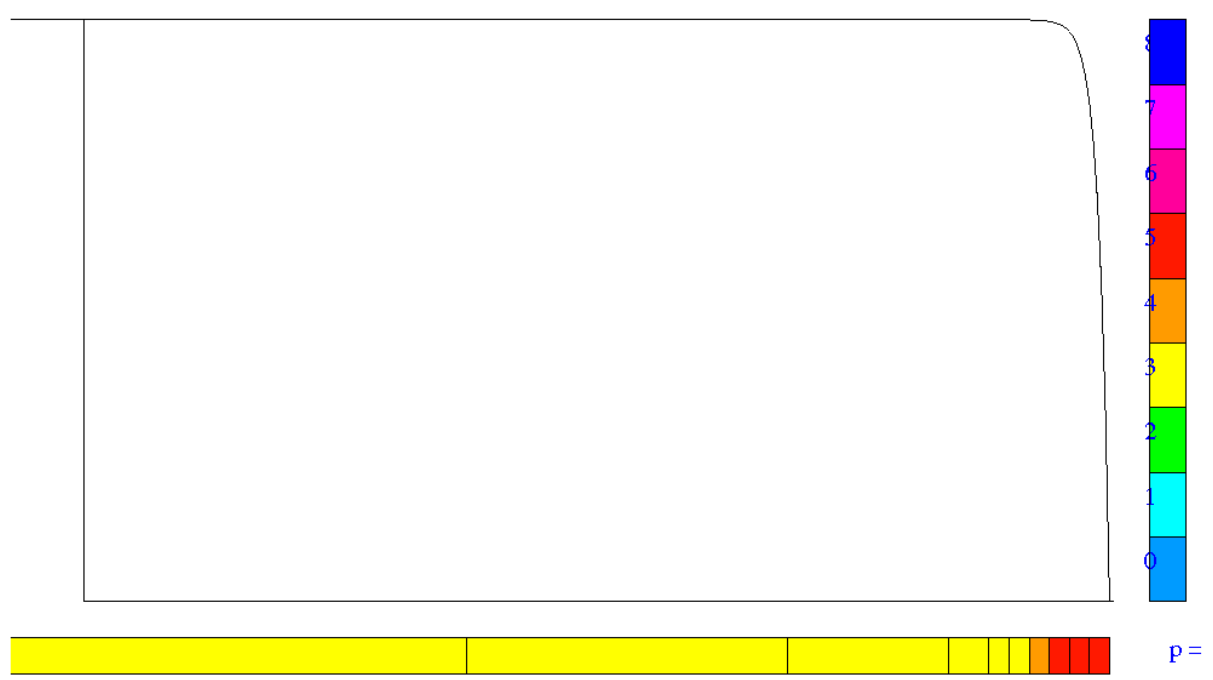

Figure 2: Diffusion parameter $\epsilon=10^{-3}$ : resolution of the boundary layer; exact solution $u$ and numerical solution $u_{h p}$ overlap with each other.

approximate eigenvectors of the 1D Laplacian (with Neumann boundary conditions),

$$
\left\{\begin{array}{l}
\lambda_{n} \in \mathbb{R}, \phi_{n} \in \mathcal{P}^{p+\Delta p}(0,1) \\
\int_{0}^{1} \phi_{n}^{\prime} \delta \phi^{\prime} d \xi=\lambda_{n} \int_{0}^{1} \phi_{n} \delta \phi d \xi, \quad \forall \delta \phi \in \mathcal{P}^{p+\Delta p}(0,1)
\end{array}\right.
$$




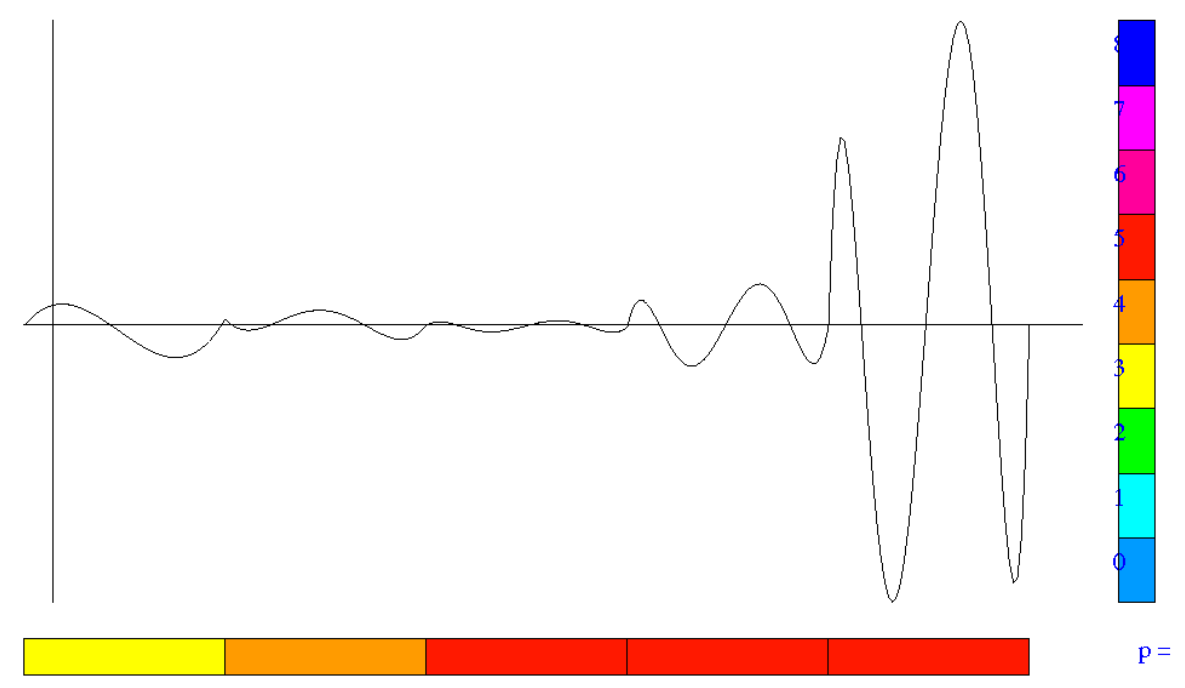

Figure 3: Diffusion parameter $\epsilon=10^{-3}$ : error representation function $\phi$.

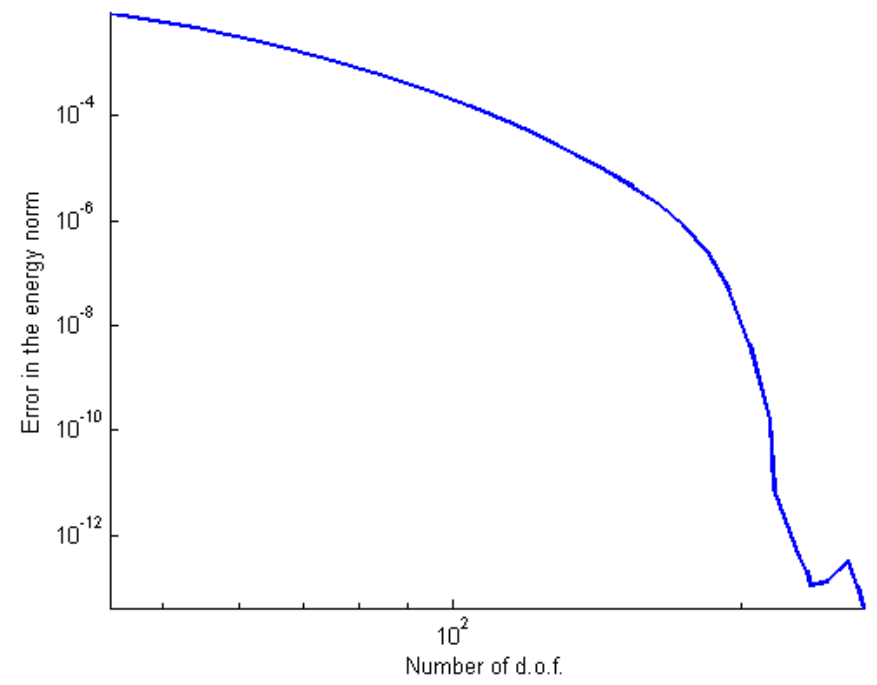

Figure 4: Diffusion parameter $\epsilon=10^{-6}$ : convergence history.

The eigenproblem above was discretized in turn with Legendre polynomials. With the use of the eigenvectors, the stiffness matrix for the local, element problems was reduced to a diagonal matrix (the off-diagonal terms were set to "clean" zeros). The diagonalization of the stiffness matrix eliminates the round off error effect from the determination of optimal test functions (equations are decoupled). Still, we have not observed any significant improvement in the global results which indicates conditioning problems with the global and not just the local stiffness matrix. 


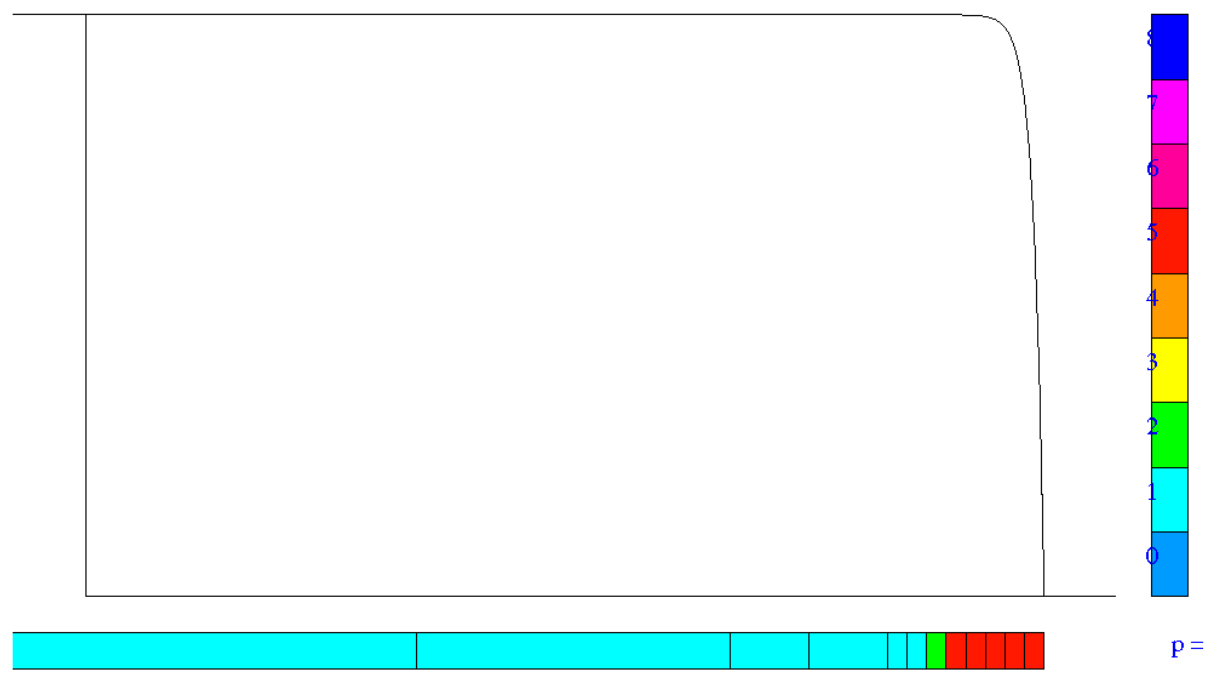

Figure 5: Diffusion parameter $\epsilon=10^{-6}$ : resolution of the boundary layer.

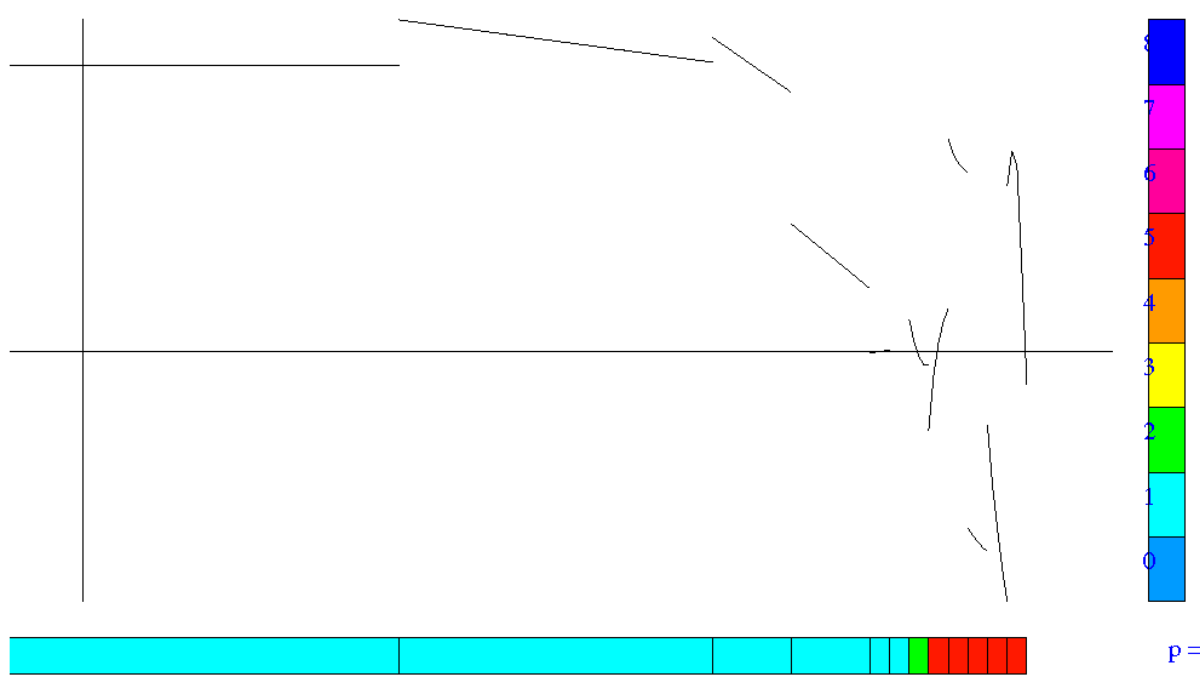

Figure 6: Diffusion parameter $\epsilon=10^{-6}$ : error representation function $\phi$.

A remedy. A simple idea that came to mind was to work with a rescaled inner product:

$$
\left(v_{k}, \delta v\right)_{k}=\int_{x_{k-1}}^{x_{k}}\left(h_{k} v_{k}^{\prime} \delta v^{\prime}+v_{k} \delta v\right) \alpha(x) d x
$$


where $h_{k}$ is the local element size. The norm is now mesh-dependent and changes with every $h$-refinement (a "moving target" strategy). The global energy error is no longer guaranteed to behave monotonically with $h$-refinements. Indeed, looking at the convergence history shown in Fig. 7 , the period corresponding to $h$-refinements is characterized with practically constant error (the numerical values display a modest decrease). Fig. 8 showing a $10^{5} \times$ zoom on the boundary layer reveals a perfect resolution of the boundary

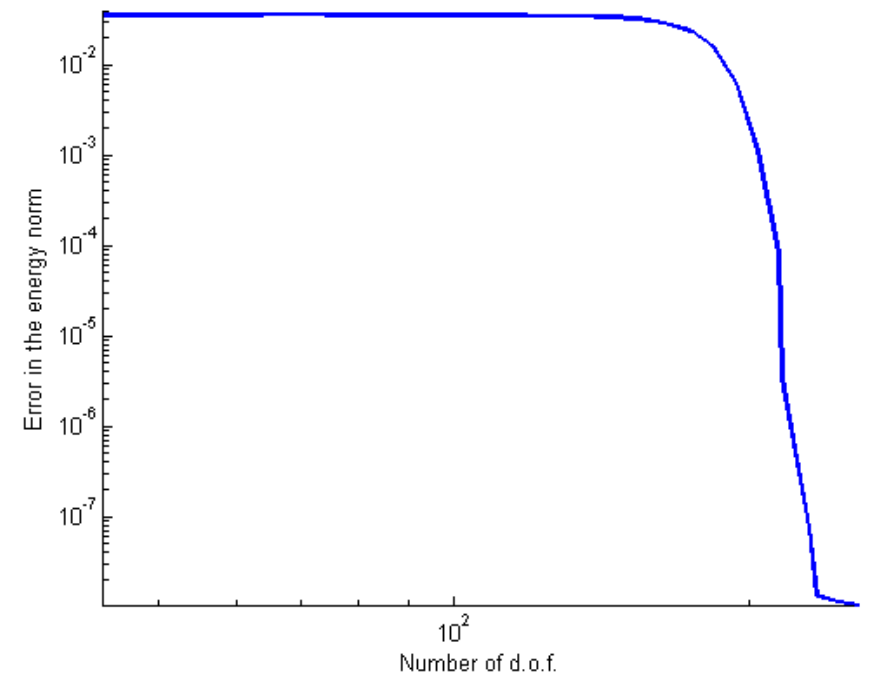

Figure 7: Diffusion parameter $\epsilon=10^{-6}$, rescaled inner product: convergence history.

layer. Most importantly, the error representation function $\phi$ shown in Fig. 9 is again globally continuous.

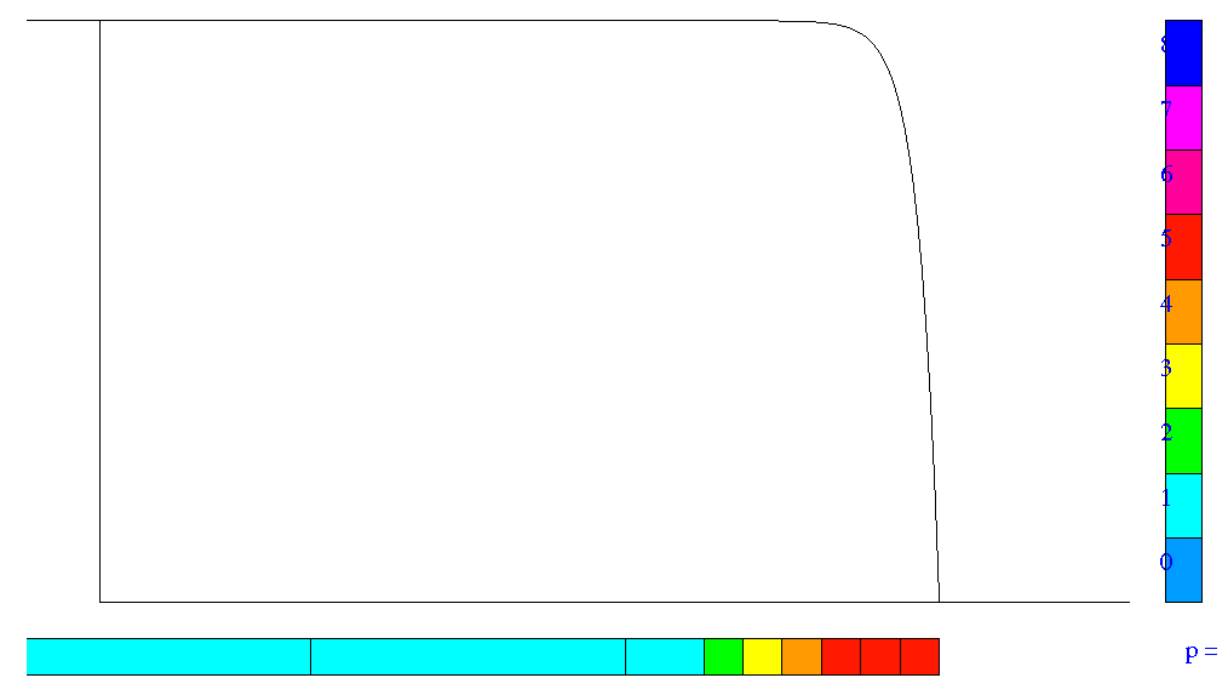

Figure 8: Diffusion parameter $\epsilon=10^{-6}$, rescaled inner product: resolution of the boundary layer. 


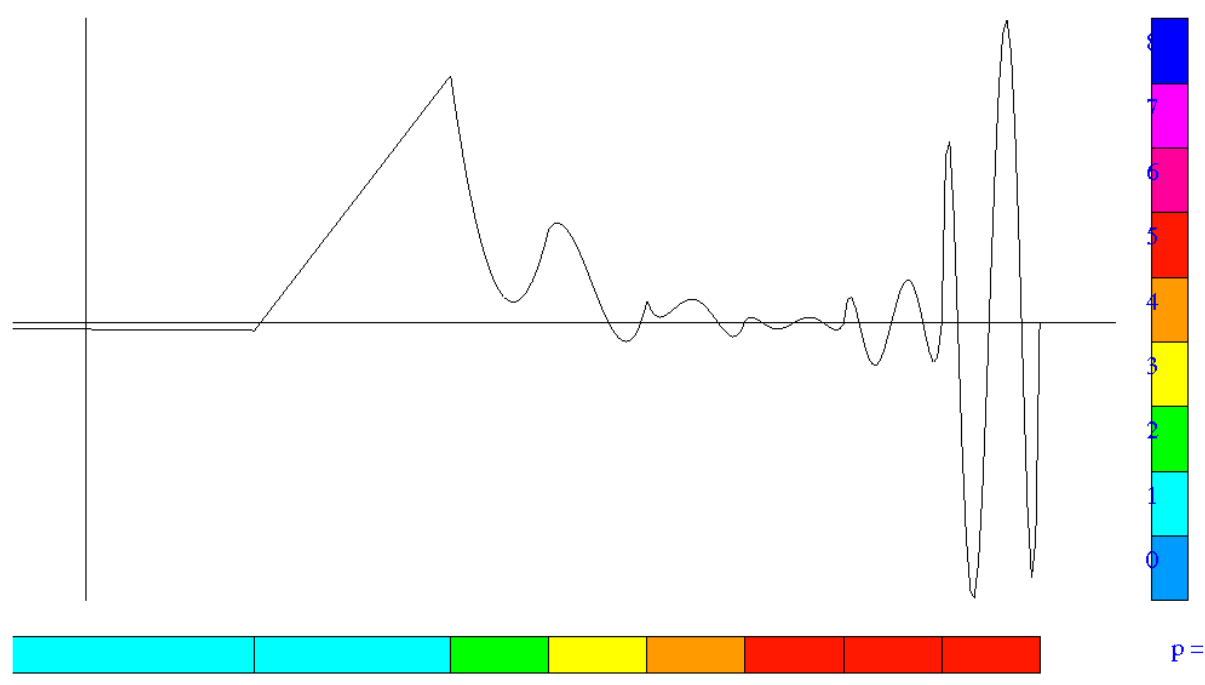

Figure 9: Diffusion parameter $\epsilon=10^{-6}$, rescaled inner product: error representation function $\phi$.

The minimum value of $\epsilon$, we have been able to solve for with the rescaled inner product, is $\epsilon=10^{-11}$. We have tried to increase the power of the element size $h_{k}$ in (3.55) beyond one. It looks like the method does work with an exponent greater than one (e.g. $1.1-1.3$ ) but falls apart when the exponent equals 2. As practical range of interest is $\epsilon \geq 10^{-7}$, we have been content with the use of (3.55).

Remark 1 All numerical results presented in this and subsequent sections were carried out using our $h p \mathrm{FE}$ codes supporting the whole exact sequence. In other words, the $1 \mathrm{D}$ code supports both $H^{1}$ - and $L^{2}$-conforming elements, whereas the $2 \mathrm{D}$ code supports $H^{1}$-, $\boldsymbol{H}$ (curl)- and $L^{2}$-conforming elements. The orders for elements of different conformity type are related. Corresponding to $1 \mathrm{D} H^{1}$-conforming element of order $p$ is $L^{2}$-conforming element of order $p-1$. The color code in pictures presenting meshes corresponds always to the $H^{1}$-conforming elements. This means that the actual order of approximation used for the 1D $L^{2}$-conforming elements, is one order less. Similarly, the order for quads in 2D drops by one as we move from $H^{1}$ to $L^{2}$-conforming elements. However, as our 2D code supports only Nédélec triangles of the second type, the order for $L^{2}$-conforming triangles is always $p-2$, with $p$ being the order of the corresponding $H^{1}$-conforming element. For details on exact sequences and $h p$ discretizations, see [24].

\section{2D Numerical Experiments}

We consider now the 2D convection-dominated diffusion problem

$$
\left\{\begin{aligned}
\frac{1}{\epsilon} \boldsymbol{\sigma}-\nabla u & =0 & & \text { in } \Omega \\
-\operatorname{div} \boldsymbol{\sigma}+\operatorname{div}(\boldsymbol{\beta} u) & =f & & \text { in } \Omega
\end{aligned}\right.
$$

accompanied with boundary condition for velocity $u$,

$$
u=u_{0} \text { on } \partial \Omega
$$

where $\Omega \subset \mathbb{R}^{2}$, right-hand side $f$ and boundary data $u_{0}$ are given, and $\boldsymbol{\beta}$ is a prescribed advection field. 
Given an element $K$, we multiply equations (4.56) with test functions $\boldsymbol{\tau}, v$, integrate over the element, and integrate by parts to arrive at the variational formulation:

$$
\left\{\begin{array}{lrlll}
\frac{1}{\epsilon} \int_{K} \boldsymbol{\sigma} \boldsymbol{\tau} & +\int_{K} u \operatorname{div} \boldsymbol{\tau}-\int_{\partial K} \hat{u} \tau_{n} & =0 & \forall \boldsymbol{\tau} \\
\int_{K} \boldsymbol{\sigma} \nabla v-\int_{\partial K} \hat{\sigma}_{n} \operatorname{sgn}(\boldsymbol{n}) v & -\int_{K} u \boldsymbol{\beta} \cdot \nabla v+\int_{\partial K} \hat{u} \beta_{n} v & =\int_{K} f v & \forall v
\end{array}\right.
$$

where the normal stress $\hat{\sigma}_{n}$ and the velocity $\hat{u}$ have become independent unknowns on the boundary of the element. The normal stress is defined using a globally fixed normal $\boldsymbol{n}_{e}$ for element edge $e$, with function $\operatorname{sgn}(\boldsymbol{n})$ indicating consistency of global and local, element outward normal unit vectors,

$$
\operatorname{sgn}(\boldsymbol{n})=\left\{\begin{aligned}
1 & \text { if } \boldsymbol{n}=\boldsymbol{n}_{e} \\
-1 & \text { if } \boldsymbol{n}=-\boldsymbol{n}_{e}
\end{aligned}\right.
$$

Energy setting. Presence of the gradient and divergence operators acting on test functions and CauchySchwarz inequality clearly suggest the choice of the space $\boldsymbol{H}(\operatorname{div}, K)$ for $\boldsymbol{\tau}$ and $H^{1}(K)$ for $v$ as well as the spaces $\boldsymbol{L}^{2}(K)$ and $L^{2}(K)$ for the field unknowns $\boldsymbol{\sigma}$ and $u$, respectively. Recall that in 1D the fluxes are just numbers so their functional setting is rather trivial. In the multi-dimensional setting the fluxes should be in the space dual to the test functions:

$$
\prod_{K}\left(\boldsymbol{H}(\operatorname{div}, K) \times H^{1}(K)\right)
$$

Remark 2 A concrete characterization of the flux spaces was recently given in [9]. The trace $\hat{u}_{n}$ comes from space $\widetilde{H}^{1 / 2}\left(\Gamma_{h}^{0}\right)$ defined as the space of traces of functions from $H_{0}^{1}(\Omega)$ to the internal skeleton of the mesh:

$$
\Gamma_{h}^{0}=\Gamma_{h}-\partial \Omega \quad \text { where the skeleton } \Gamma_{h}=\bigcup_{K} \partial K
$$

and the flux $\hat{\sigma}_{n} \in H^{1 / 2}\left(\Gamma_{h}\right)$ where $H^{1 / 2}\left(\Gamma_{h}\right)$ denotes the space of traces of functions from $\boldsymbol{H}(\operatorname{div}, \Omega)$ to the mesh skeleton. Both spaces are equipped with the minimum energy extension norms. These facts were unknown to us at the time of writing this paper and the remark was added during the review process.

Discretization. We will compute with both triangular and quadrilateral elements, restricting ourselves to 1-irregular meshes only. Each element $K$ supports two field variables: the vector values stress $\boldsymbol{\sigma}$ and the velocity $u$. Each element edge supports two fluxes: the normal stress $\hat{\sigma}_{n}$ and the velocity $\hat{u}$. For triangles, we use the spaces $\mathcal{P}^{p}$, whereas for quads we use the spaces $\mathcal{Q}^{(p, q)}=\mathcal{P}^{p} \otimes \mathcal{P}^{q}$ with the standard relation

$$
\phi(\boldsymbol{x}):=\hat{\phi}(\boldsymbol{\xi}), \quad \boldsymbol{x}=\boldsymbol{x}_{K}(\boldsymbol{\xi})
$$

between the master and physical element shape functions. Here $\boldsymbol{x}_{K}$ stands for the element map transforming the corresponding master triangle or square into the physical element. The logic of exact sequence and $L^{2}-$ conforming discretizations suggests a slightly different relation between the physical element shape functions $\phi(\boldsymbol{x})$ and the master element shape functions $\hat{\phi}(\boldsymbol{\xi})[24]$

$$
\phi(\boldsymbol{x}):=\frac{1}{j} \hat{\phi}(\boldsymbol{\xi}), \quad \boldsymbol{x}=\boldsymbol{x}_{K}(\boldsymbol{\xi})
$$

where $j$ denotes the jacobian for the element map, $j=\operatorname{det}\left(\nabla \boldsymbol{x}_{K}\right)$. However, all presented numerical examples will involve affine element only, for which the difference between the two relations reduces to a local scaling with the constant jacobian only. Element order of approximation $p$ for triangles and $(p, q)$ for quads can vary locally, from element to element. 
Discretization for fluxes is fixed using a maximum rule. For two neighboring triangles of order $p_{1}$ and $p_{2}$, the order for the shared edge is set to

$$
p_{e}=\max \left\{p_{1}, p_{2}\right\}+1
$$

There is no particularly strong reason for raising the order for the edge by one. It is mostly a legacy issue reflecting our initial experience with the DPG method for pure convection problems [8]. In the case of hanging nodes and an edge with two small neighbors on one side and equal size neighbor on the other, the discretization for the flux is chosen to be piecewise polynomial with the order set to the maximum of orders for all three neighboring elements,

$$
p_{e}=\max \left\{p_{1}, p_{2}, p_{3}\right\}+1
$$

In that sense the maximum rule applies here to both element order and size.

We use the same principle for quadrilateral meshes accounting for the directionality of approximation. This time, again for no deep reason, we chose to set the order of fluxes just to the maximum of the directional orders for the neighboring elements,

$$
p_{e}=\max \left\{q_{1}, q_{2}, q_{3}\right\}
$$

Here $q_{i}$ is the order of discretization of the $i$-th neighbor in the direction of the edge.

For each element trial shape function and for each edge trial shape functions, we determine the optimal test function by solving the local problem,

$$
\left\{\begin{array}{l}
\tau_{K} \in \boldsymbol{V}(K), v_{K} \in V(K) \\
\left(\boldsymbol{\tau}_{K}, \boldsymbol{\delta} \boldsymbol{\tau}\right)_{H(\operatorname{div})}+\left(v_{K}, \delta v\right)_{H^{1}}=b_{K}\left(\left(\boldsymbol{\sigma}, u, \hat{\sigma}_{n}, \hat{u}\right),(\boldsymbol{\delta} \boldsymbol{\tau}, \delta v)\right) \quad \forall \boldsymbol{\delta} \tau \in \boldsymbol{V}(K), \delta v \in V(K)
\end{array}\right.
$$

where $\boldsymbol{V}(K), V(K)$ are the enriched spaces: $\mathcal{P}^{p+\Delta p}$ for triangular elements, and $\mathcal{P}^{p+\Delta p} \otimes \mathcal{P}^{q+\Delta p}$ for quads. At this point we have ignored $H$ (div)-conforming elements and approximated components of $\tau$ and $\boldsymbol{\delta} \tau$ with polynomials of equal order. Despite the piecewise polynomial fluxes for 1-irregular meshes, we have not experimented yet with piecewise polynomials for the enriched spaces corresponding to a local $h$-refinement but intend to do so in future work. The optimal test functions corresponding to the field variables $\boldsymbol{\sigma}, u$ span over one element only whereas the optimal test functions corresponding to the fluxes $\hat{\sigma}_{n}, \hat{u}$ span over the elements neighboring the particular edge. Fluxes $\hat{u}$ on the boundary of the domain are determined by performing $L^{2}$-projections of the corresponding boundary data onto the corresponding trial space.

Finally, we start our experiments with weighted inner products for the $\boldsymbol{H}(\operatorname{div})$ and $H^{1}$ spaces,

$$
\begin{aligned}
(\boldsymbol{\tau}, \boldsymbol{\delta} \boldsymbol{\tau})_{H(\operatorname{div})} & =\int_{K}\{\operatorname{div} \boldsymbol{\tau} \operatorname{div} \boldsymbol{\delta} \boldsymbol{\tau}+\boldsymbol{\tau} \boldsymbol{\delta} \boldsymbol{\tau}\} w(\boldsymbol{x}) d \boldsymbol{x} \\
(v, \delta v)_{H^{1}} & =\int_{K}\{\nabla v \nabla \delta v+v \delta v\} w(\boldsymbol{x}) d \boldsymbol{x}
\end{aligned}
$$

The same setting is used to determine the element error representation functions $\left(\boldsymbol{\psi}_{K}, \phi_{K}\right)$.

All 2D numerical experiments reported in this paper were done for a unit square domain $\Omega=(0,1)^{2}$, constant advection vector $\boldsymbol{\beta}$, and boundary conditions defined in Fig. 10. The weight $w(\boldsymbol{x})$ for the inner products (4.67) was selected following our 1D experience and is shown in Fig. 11. In $2 \mathrm{D}$ the situation is a bit more difficult than in $1 \mathrm{D}$ as the inflow boundary meets the no-flow boundary at the north-west and south-east corners. The choice of the weight reflects the intention of emphasizing the inflow boundary over the no-flow boundary in the resulting DPG energy norm. Our numerical experiments focus again on determining the minimum values of the diffusion parameter $\epsilon$ for which we can solve the problem. The global system of equations was solved using a frontal solver for symmetric problems (with no pivoting). The element d.o.f. were ordered as follows: field variables first, fluxes next. This effectively results in a static condensation of the interior d.o.f. and improves the conditioning of the global matrix. 


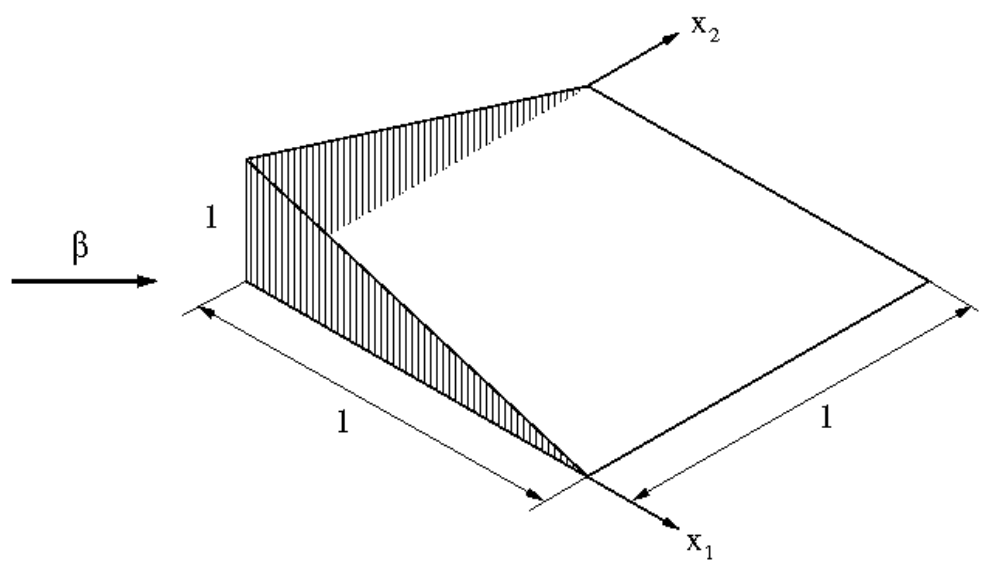

Figure 10: Convection-dominated diffusion in 2D: boundary conditions

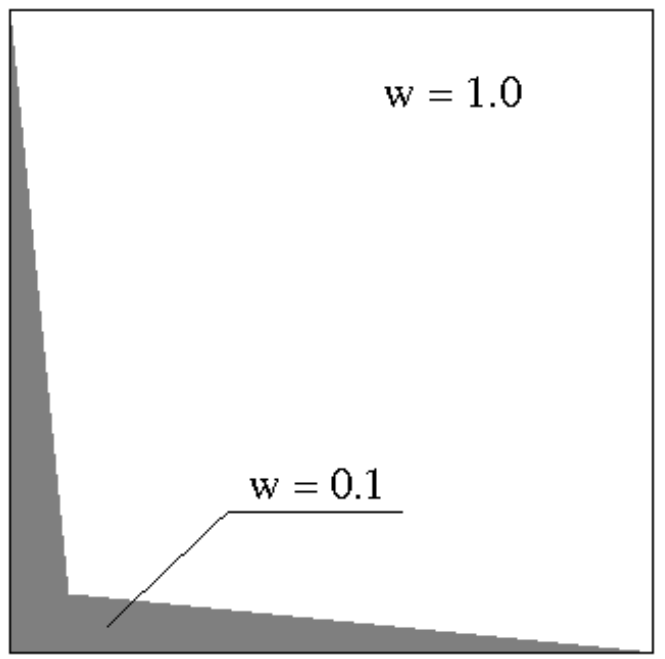

Figure 11: Convection-dominated diffusion in 2D: definition of the weight. 


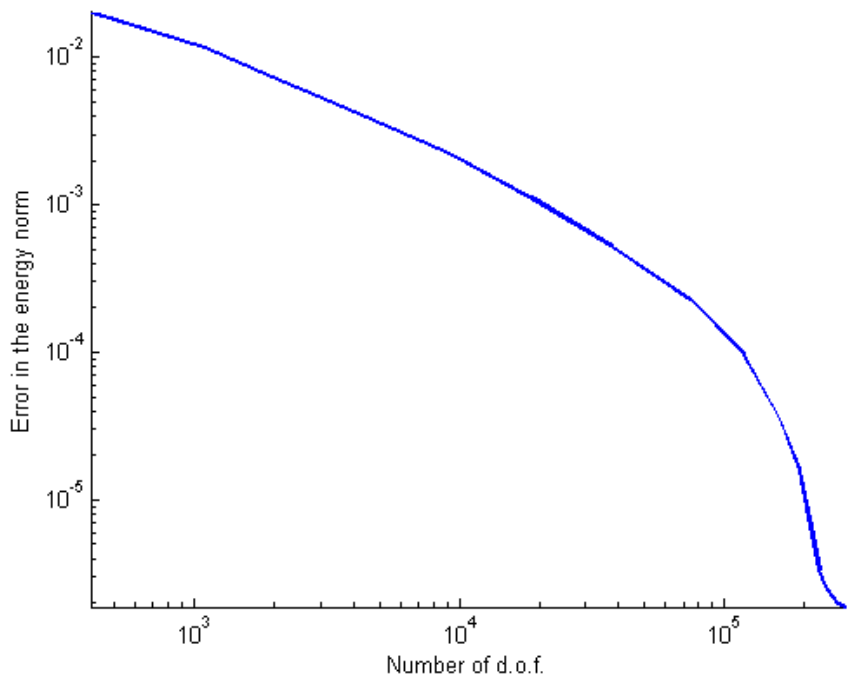

Figure 12: Diffusion parameter $\epsilon=10^{-3}$ : convergence history for triangular meshes.

\section{Triangular Meshes}

The advection vector was set to $\boldsymbol{\beta}=(1,2)$ in order to avoid alignment with the mesh. ${ }^{2}$ We have used the same adaptive algorithm as for the $1 \mathrm{D}$ problems. The increment in the polynomial order was set to $\Delta p=4$, and the maximum order was limited to $p_{\max }=4$. The "working horse" are elements of second order and our inital mesh always consists of eight elements with $p=2$. We begin with a modest case with $\epsilon=10^{-3}$. Fig.12 presents convergence history on the $\log$-log scale. The maximum number of d.o.f. after 21 iterations reached a total of 284,287 and the energy norm was reduced by two orders of magnitude. Convergence is monotone throughout the whole process. Fig. 13 presents the final mesh. The inflow conditions are non-smooth at the south-west corner which causes a visible diffusion along the streamline picked by the refinements. Figures 14 and 15 present $10^{2} \times$ zooms on the mid-point of the north edge and the north-east corner showing the $h p$-meshes generated by the algorithm. Figures 16 through 20 present the corresponding resolution of the flow and the boundary layers, with and without the mesh. The visible mesh structure on the contour plots without the mesh indicates still a non-perfect resolution. The computed solution range is $(-0.81043 E-01,0.10783 E+01)$.

Solution for $\epsilon=10^{-4}$ was possible but at the limit of the four year old IBM ThinkPad on which the presented experiments were made. The final mesh reached almost $1 \mathrm{M}$ d.o.f. and, in order to avoid the round-off breakdown, we had to rescale the mass terms in the inner product by a factor of 10. Clearly, triangular meshes and isotropic refinements are not the way to go when it comes to boundary layers.

\section{Quadrilateral Meshes}

Each quadrilateral element is assigned an isotropy flag which is fixed upon solving the local problem for determining the error representation functions $\left(\boldsymbol{\psi}_{K}, \phi_{K}\right)$,

$$
\left\{\begin{array}{r}
\boldsymbol{\psi}_{K} \in \boldsymbol{V}(K), \phi_{K} \in V(K) \\
\left(\left(\boldsymbol{\psi}_{K}, \phi_{K}\right),(\delta \boldsymbol{\tau}, \delta v)\right)_{K}=b_{K}\left(\left(\boldsymbol{\sigma}_{h p}, u_{h p}, \hat{\sigma}_{n}, \hat{u}\right),(\delta \boldsymbol{\tau}, \delta v)\right)-l_{K}((\delta \boldsymbol{\tau}, \delta v)) \\
\forall \delta \boldsymbol{\tau} \in \boldsymbol{V}(K), \delta v \in V(K)
\end{array}\right.
$$

\footnotetext{
${ }^{2}$ The problem then would have been easier.
} 

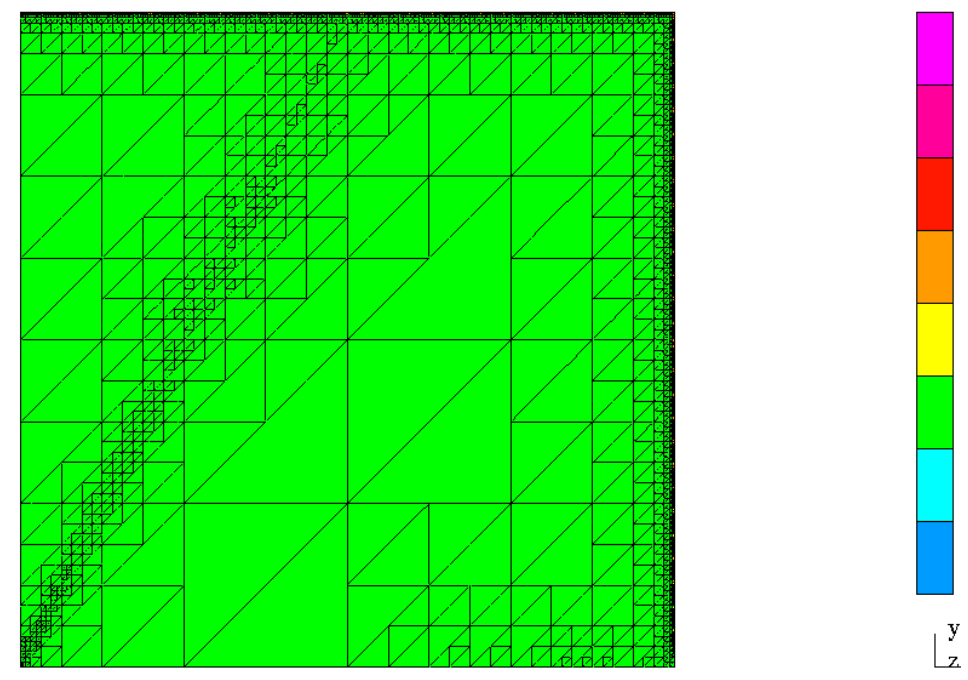

Figure 13: Diffustion parameter $\epsilon=10^{-3}$ : final mesh after 21 iterations.

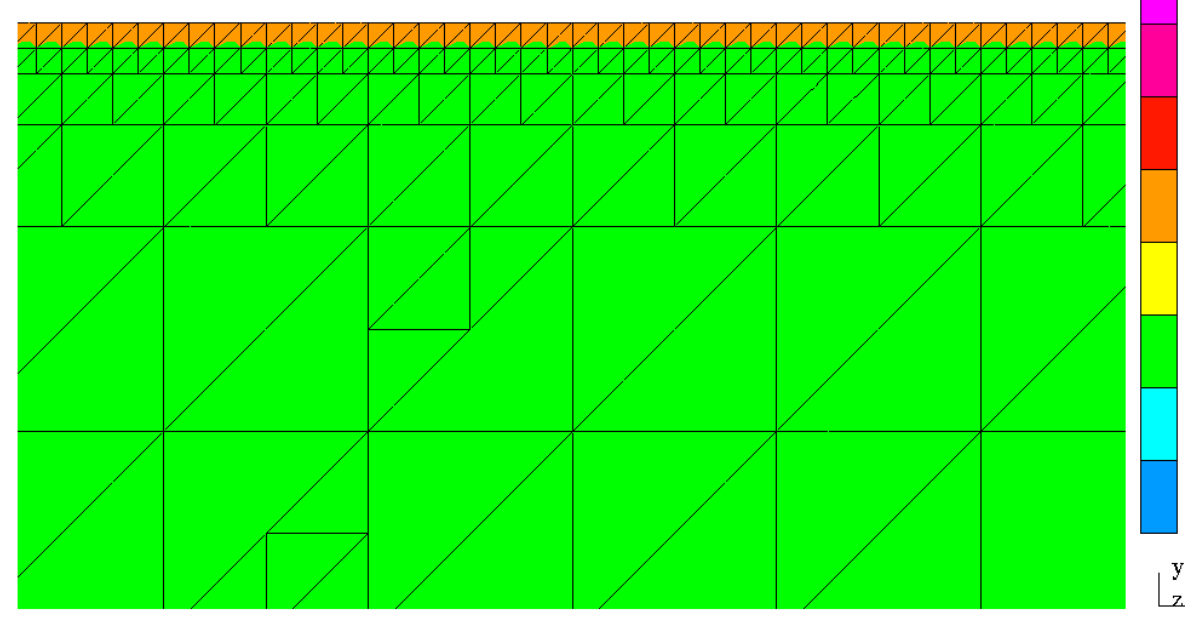

Figure 14: Diffusion parameter $\epsilon=10^{-3}$ : final mesh after 21 iterations, $10^{-2} \times$ zoom on upper boundary. 


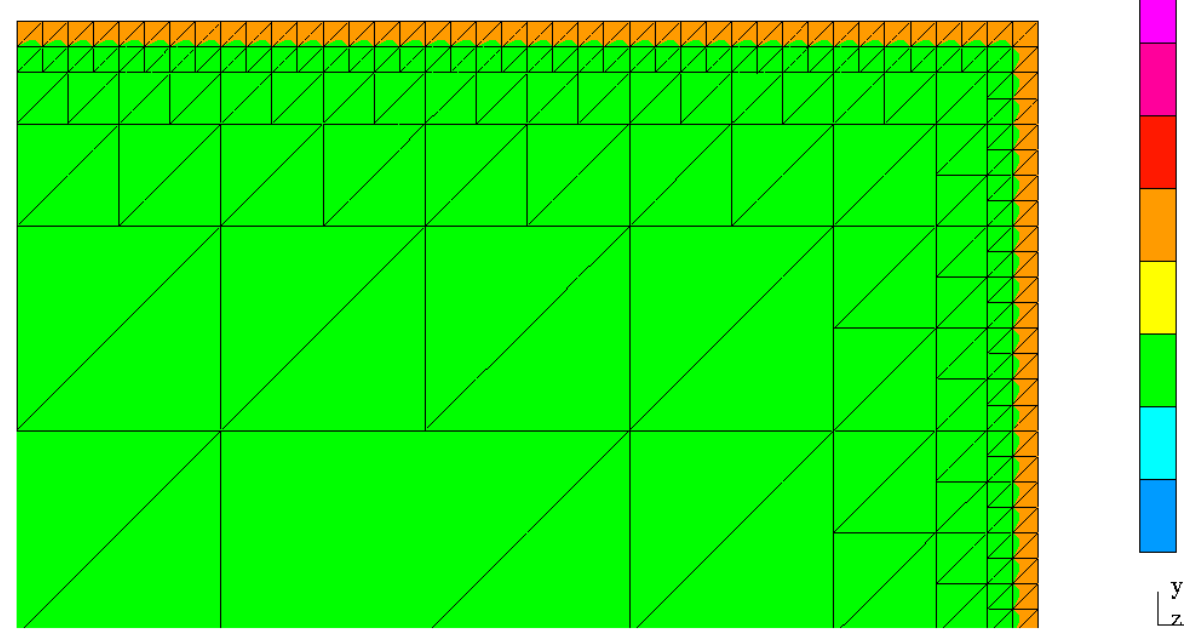

Figure 15: Diffusion parameter $\epsilon=10^{-3}$ : final mesh after 21 iterations. $10^{-2} \times$ zoom on north-east corner.
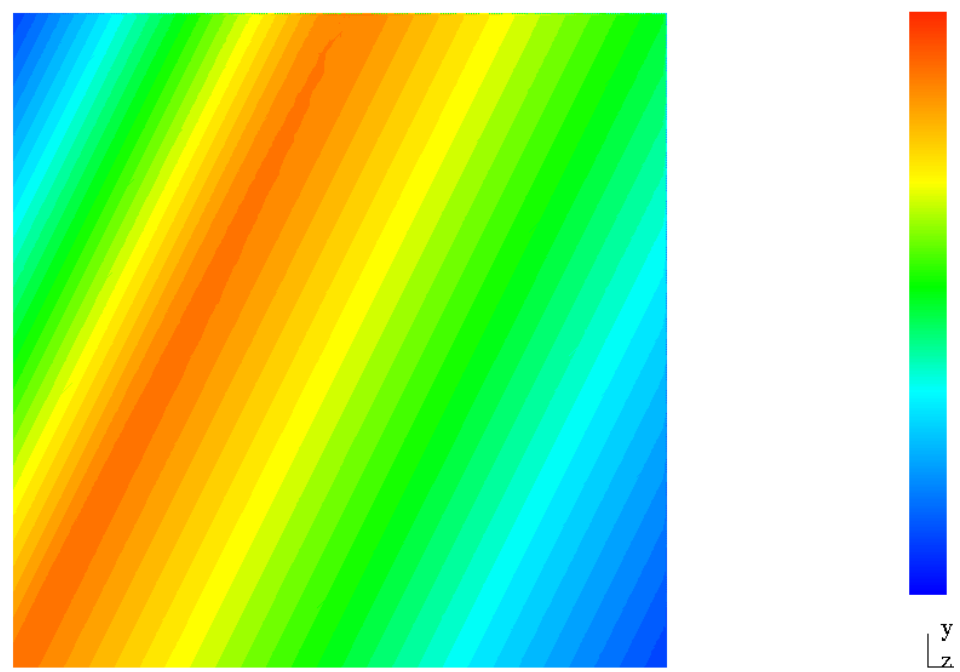

Figure 16: Diffusion parameter $\epsilon=10^{-3}$ : solution $u$. 


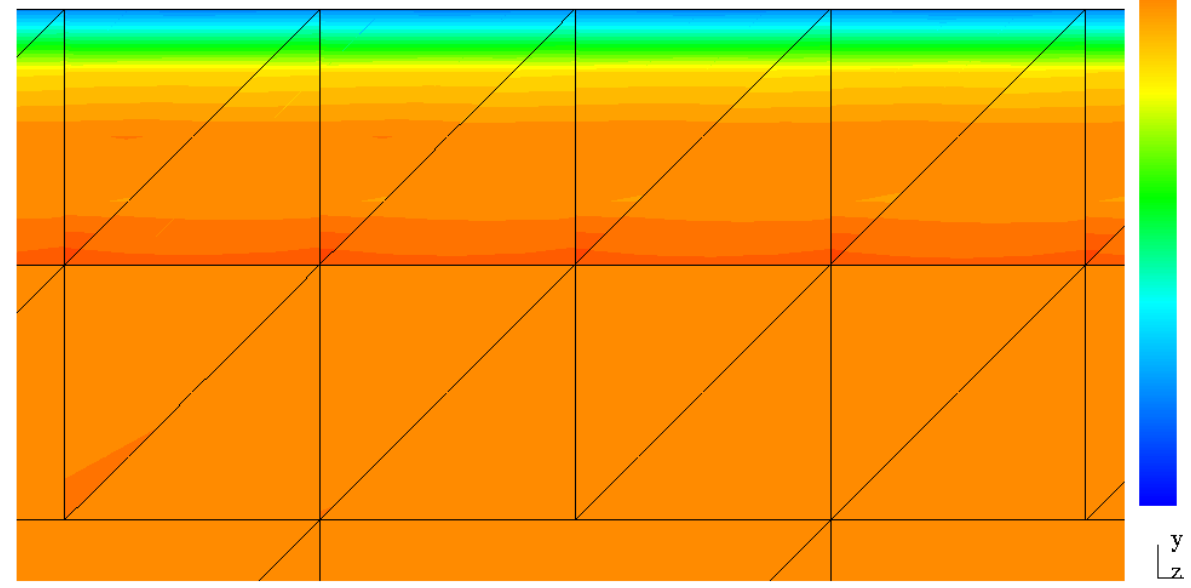

Figure 17: Diffusion parameter $\epsilon=10^{-3}$ : solution $u, 10^{2} \times$ zoom on top boundary with the mesh.

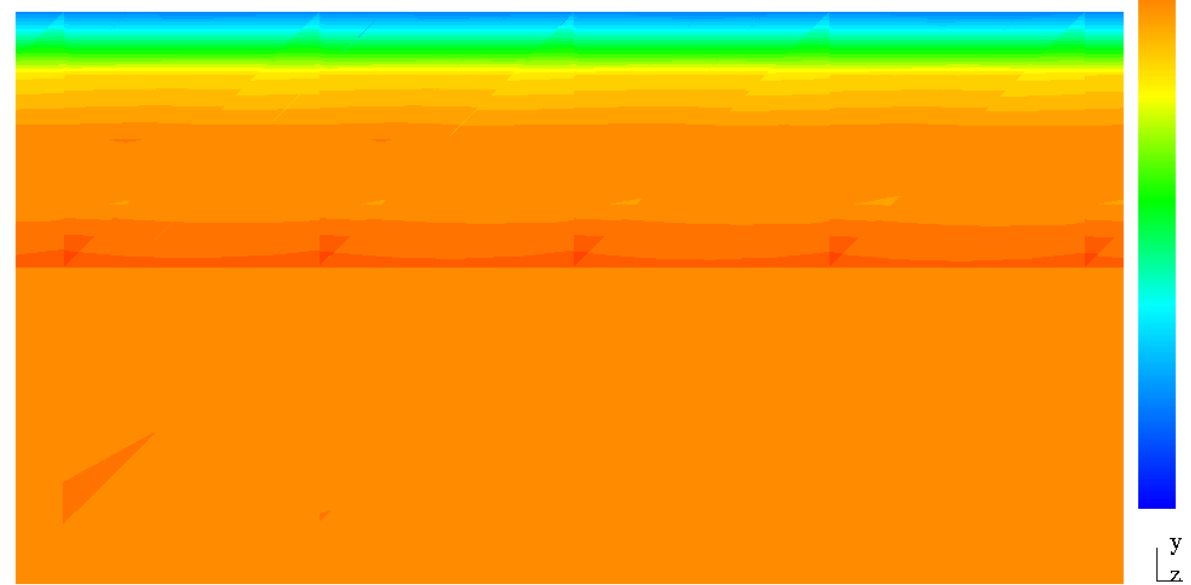

Figure 18: Diffusion parameter $\epsilon=10^{-3}$ : solution $u, 10^{2} \times$ zoom on top boundary without the mesh. 

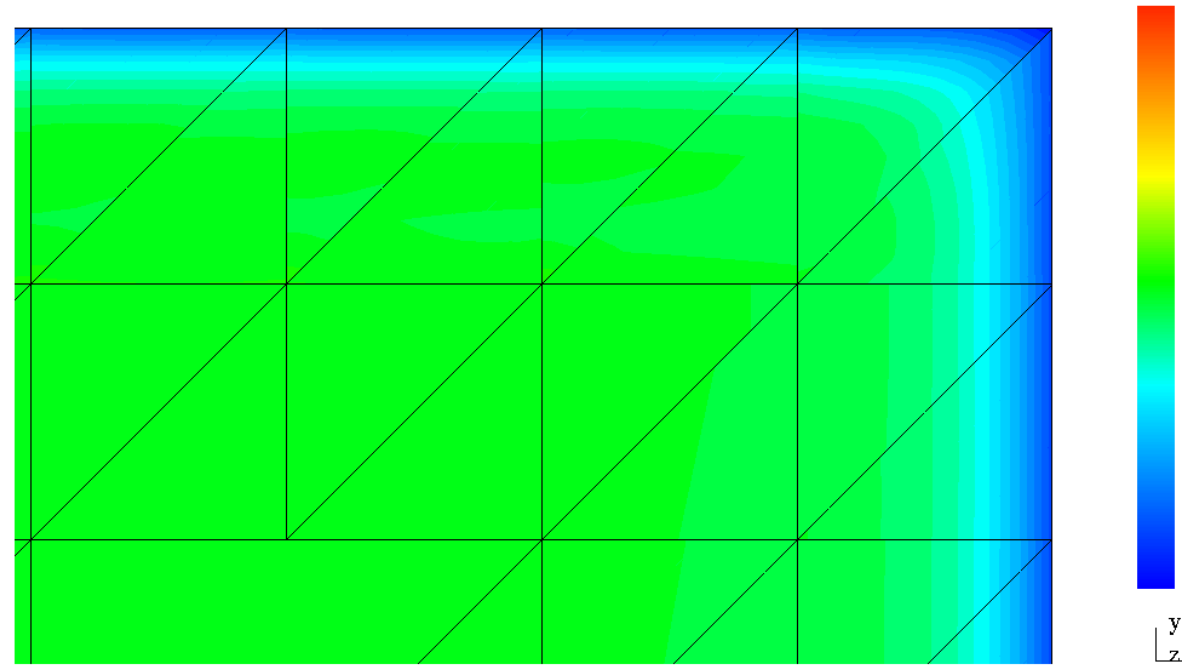

Figure 19: Diffusion parameter $\epsilon=10^{-3}$ : solution $u, 10^{2} \times$ zoom on north-east corner with the mesh.

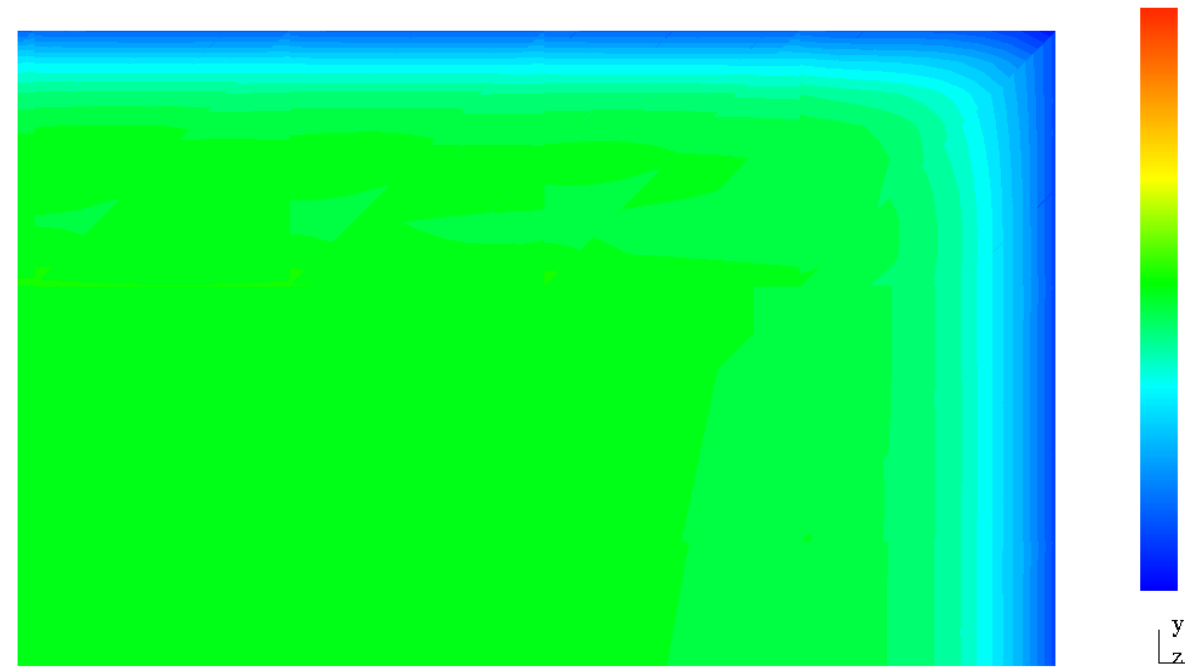

Figure 20: Diffusion parameter $\epsilon=10^{-3}$ : solution $u, 10^{2} \times$ zoom on north-east corner without the mesh. 
Here $\left(\boldsymbol{\sigma}_{h p}, u_{h p}, \hat{\sigma}_{n}, \hat{u}\right)$ is the FE solution in the element, $b_{K}$ is the element bilinear form, $(\cdot, \cdot)_{K}$ is the inner product for the test functions, and $\boldsymbol{V}(K), V(K)$ are the enriched spaces for determining the approximate optimal test functions and the error representation functions. Once the functions $\left(\boldsymbol{\psi}_{K}, \phi_{K}\right)$ are known, we define two directional contributions to the element error:

$$
c_{1}:=\int_{K}\left(\left|\psi_{K, 1}\right|^{2}+\left|\frac{\partial \phi_{K}}{\partial x_{1}}\right|^{2}\right) w(x) d x \quad c_{2}:=\int_{K}\left(\left|\psi_{K, 2}\right|^{2}+\left|\frac{\partial \phi_{K}}{\partial x_{2}}\right|^{2}\right) w(x) d x
$$

The anisotropy flag is defined now as follows:

$$
\text { Anisotropy flag }= \begin{cases}10 & \text { if } c_{1} \geq 10 c_{2} \\ 01 & \text { if } c_{2} \geq 10 c_{1} \\ 11 & \text { otherwise }\end{cases}
$$

with the choice of factor 10 being arbitrary. The elements are refined according to their isotropy flags. If the flag points in one direction only, the element is $h$ - or $p$-refined only in this direction. The formal algorithm is a slight modification of the algorithm for the triangular meshes and looks as follows:

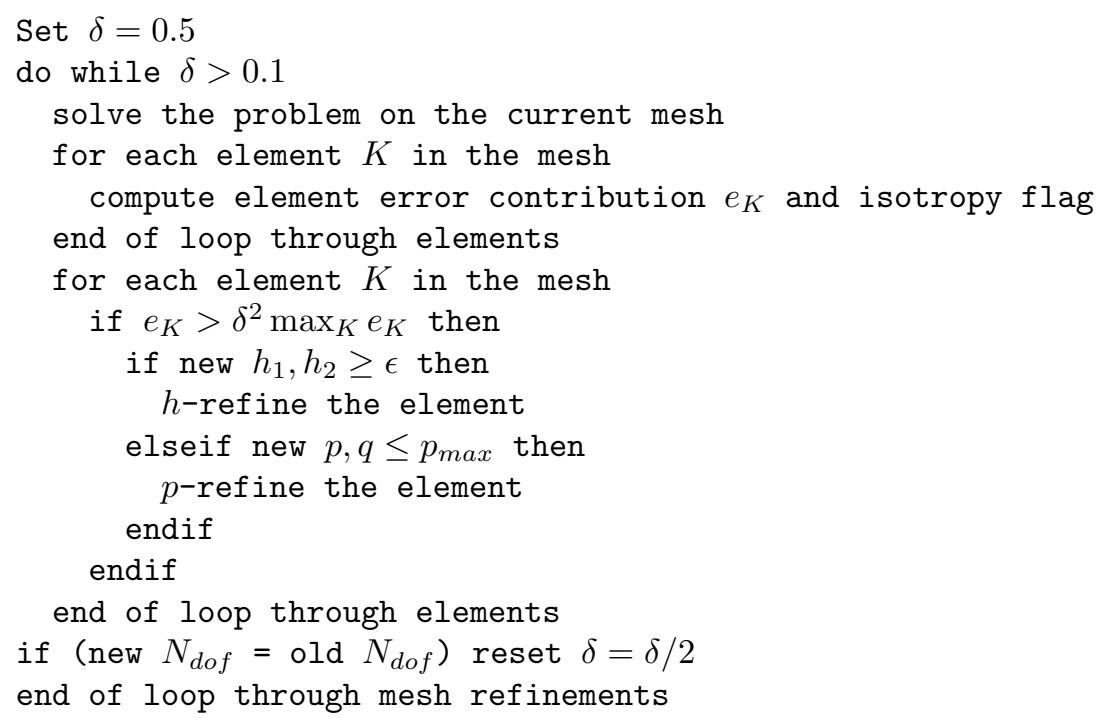

The ratio of maximum to minimum element size was limited to 10,000 . This number was determined experimentally in the process of determining the minimum diffusion parameter $\epsilon$ for which we can solve the problem inspite of the conditioning problems. In the experiments with rectangular meshes, the advection vector was changed to $\boldsymbol{\beta}=(1,1)$. The corresponding solution is then symmetric along the diagonal so that any loss of symmetry in the approximation points to either round off or programming errors.

Use of anisotropic refinements results in dramatic savings in terms of number of d.o.f. Fig. 21 presents the convergence history for $\epsilon=10^{-4}$. In place of the nearly $1 \mathrm{M}$ d.o.f. required for the triangles, the maximum number of d.o.f. reached only 134k. After 27 refinements, the energy error is reduced by four orders of magnitude (displayed is the error squared). As in the 1D examples, the error decreases monotonically. Slightly non-monotone behavior of the error in the last three iterations indicates that we are at the limit of machine precision. Quality of the solution obtained with the $h p$-refinements is illustrated in Fig. 22 displaying the solution in the north-east corner of the square domain. The range for the computed solution $u$ is $(-0.19044 E-04,0.99980 E+00)$.

We have managed to solve the problem with $\epsilon=10^{-5}$ by using additional restrictions on the element aspect ratio (to 100) and rescaling the mass terms in the norm for the test functions by a factor of 10 . However, limitation of the aspect ratio to 100 blowed up the number of d.o.f. to over 700k. 


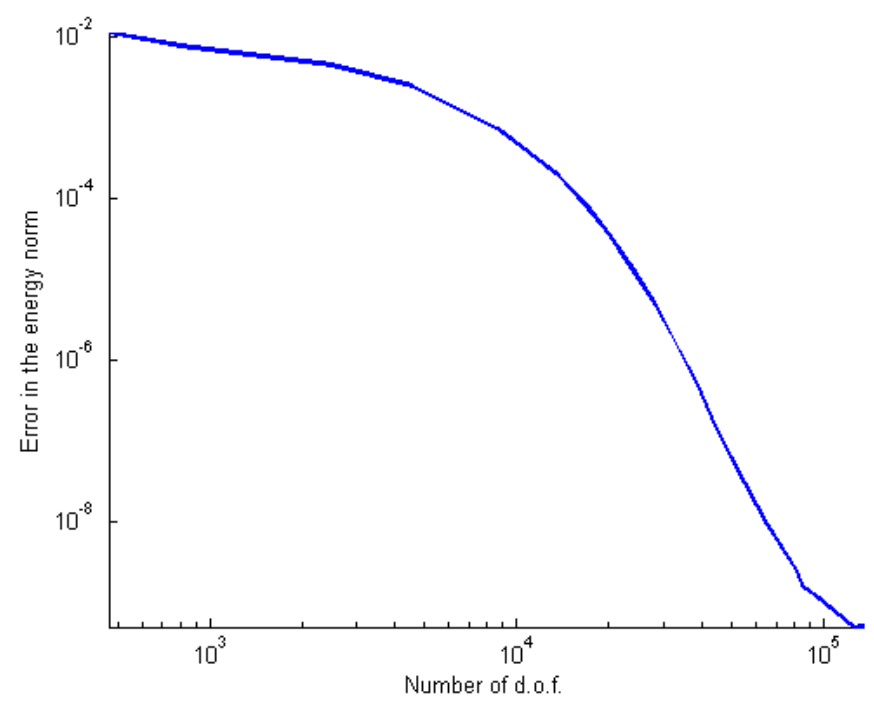

Figure 21: Diffusion parameter $\epsilon=10^{-4}$ : convergence history.

As in 1D, it is not just the local problem that is affected by the round off error effects. We have implemented the solution of local problems using a quadruple arithmetic ${ }^{3}$ with little effect on the final results.

The key point in "pushing the envelope" turned out to be the rescaling of inner product also here. The new, mesh-dependent, rescaled norm for the test functions was defined as

$$
\begin{aligned}
\|(\boldsymbol{\tau}, v)\|_{K}^{2} & =\int_{K}\left\{\left|\sqrt{h_{1}} \frac{\partial \tau_{1}}{\partial x_{1}}+\sqrt{h_{2}} \frac{\partial \tau_{2}}{\partial x_{2}}\right|^{2}+\left|\tau_{1}\right|^{2}+\left|\tau_{2}\right|^{2}\right. \\
& \left.+h_{1}\left|\frac{\partial v}{\partial x_{1}}\right|^{2}+h_{2}\left|\frac{\partial v}{\partial x_{2}}\right|^{2}+|v|^{2}\right\} w(x) d x
\end{aligned}
$$

With the rescaled inner product, we were able to solve the problem for $\epsilon=10^{-7}$. Fig. 23 shows the corresponding convergence history. The convergence is no longer monotone as the error increases slightly during the $h$-refinements and decreases only when the $p$-refinements begin. The results for $\epsilon=10^{-7}$ are illustrated in Figures 24-29. The last picture shows that there is still some room for improvement as one can detect the trace of the mesh on the contour plot. The range for the computed solution $u$ is $(-0.65259 E-06,0.99980 E+00)$.

\section{Conclusions}

A summary. Construction of stable discretizations has been at the heart of the numerical analysis since the inception of the field. The phrase "discrete stability and approximability imply convergence" is known to every numerical analyst. Equally known fact is that, except for the nice but small class of coercive problems, stability at the continuous level does not imply the discrete stability of Bubnov-Galerkin approximations. The DPG method with optimal test functions breaks through this barrier - continuous stability implies automatically discrete stability for any system of linear PDEs. In context of $h p$-discretizations, this implies automatically uniform stability in terms of the polynomial order. The difficulty is, however, shifted to

\footnotetext{
${ }^{3}$ The computation time slowed down from minutes to hours. With the current hardware, the quadruple precision does not seem to present a practical solution to round off error related problems.
} 


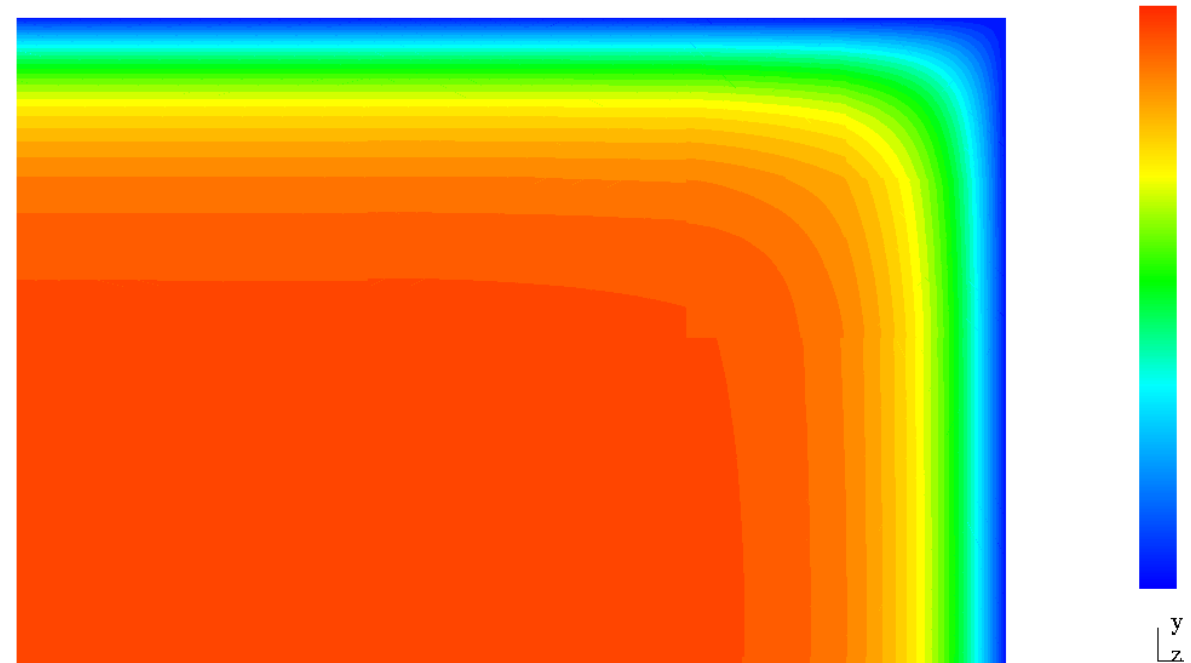

Figure 22: Diffusion parameter $\epsilon=10^{-4}$ : solution $u, 10^{3} \times$ zoom on the north-east corner.

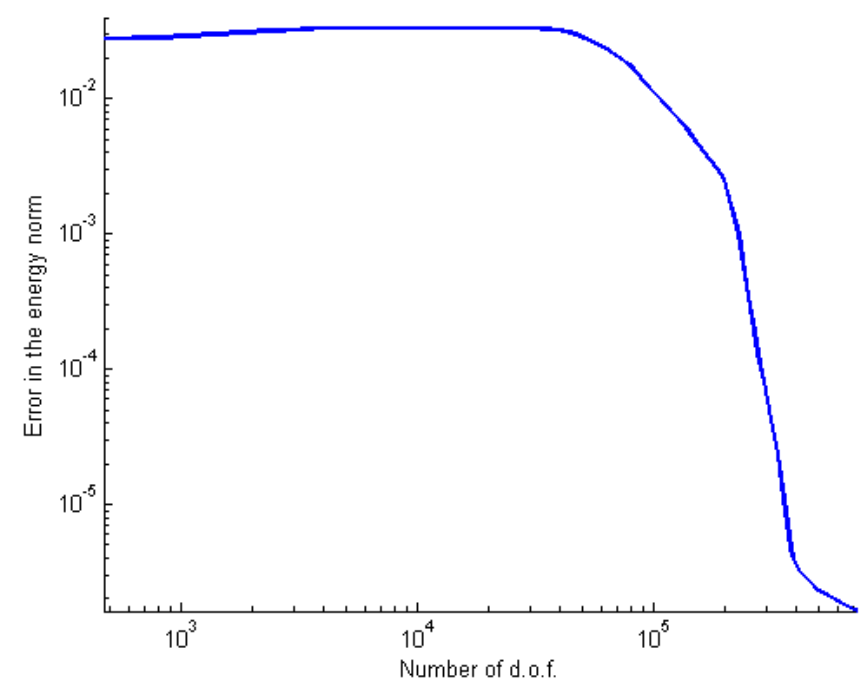

Figure 23: Diffusion parameter $\epsilon=10^{-7}$ : convergence history. 

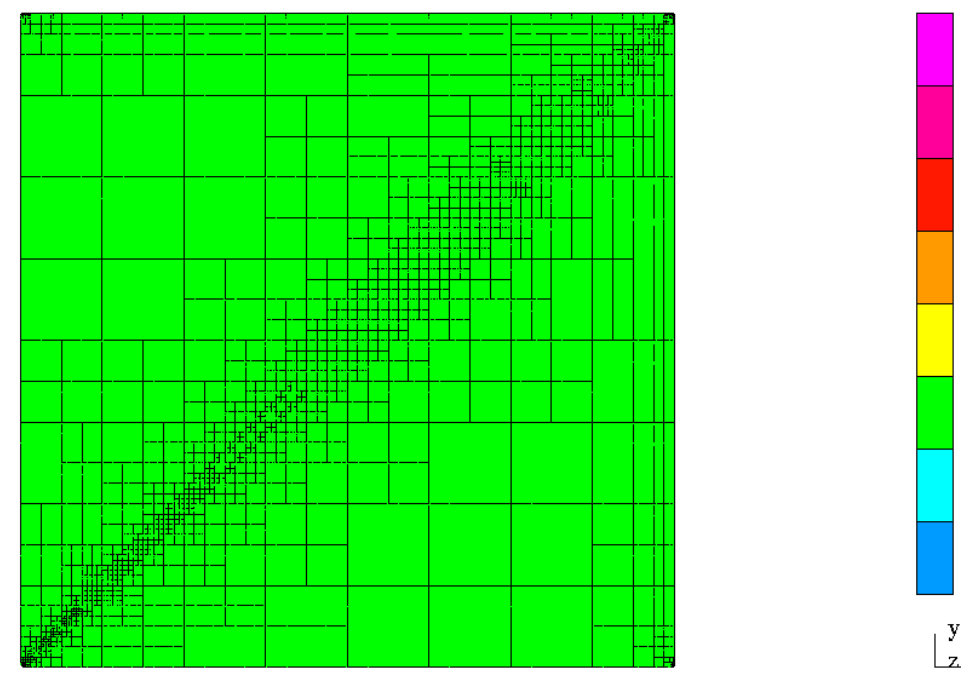

Figure 24: Diffusion parameter $\epsilon=10^{-7}: h p$ mesh after 45 mesh refinements.
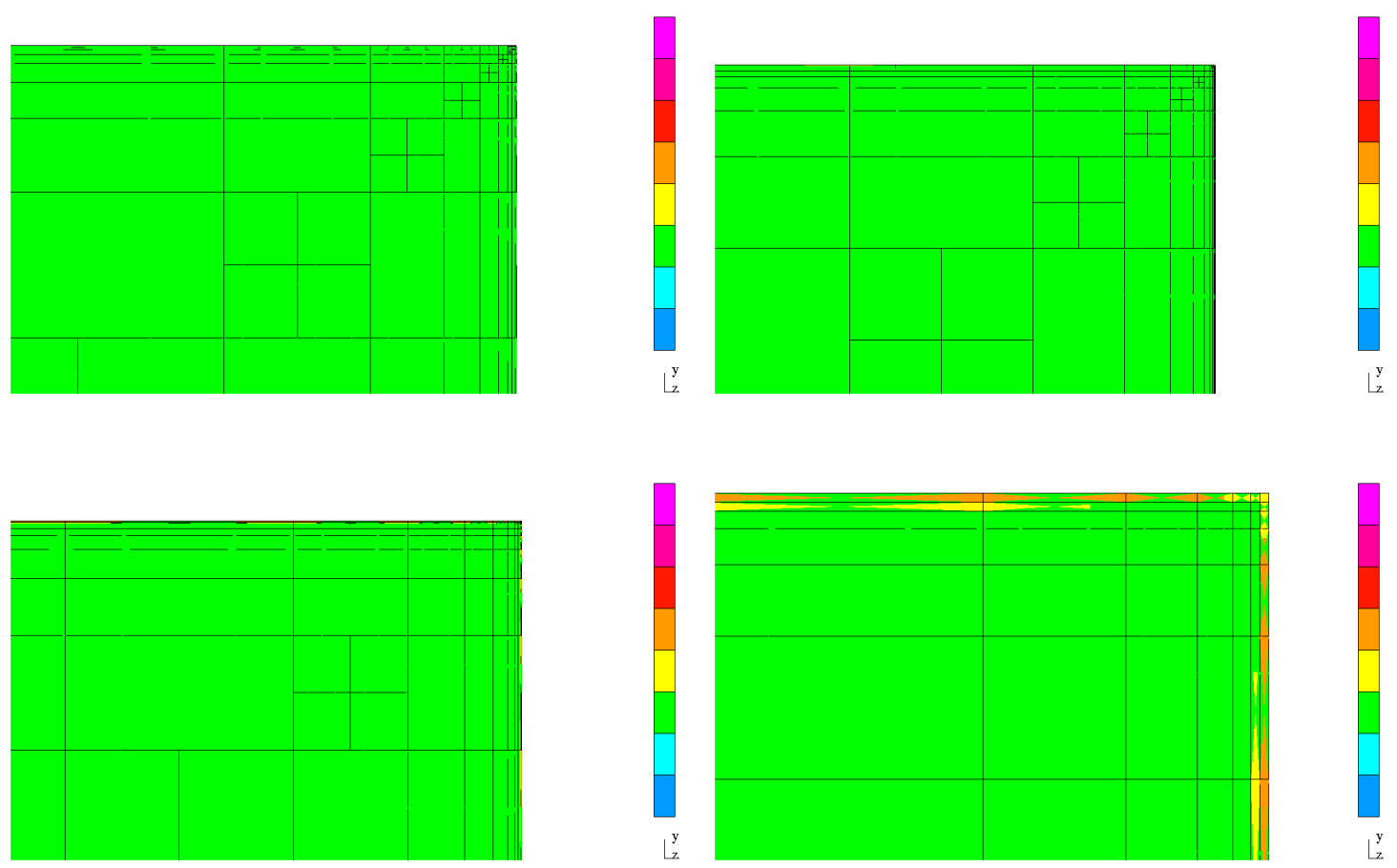

Figure 25: Diffusion parameter $\epsilon=10^{-7}: h p$ mesh after 45 mesh refinements, $10^{2}-10^{5} \times$ zooms on the north-east corner. 

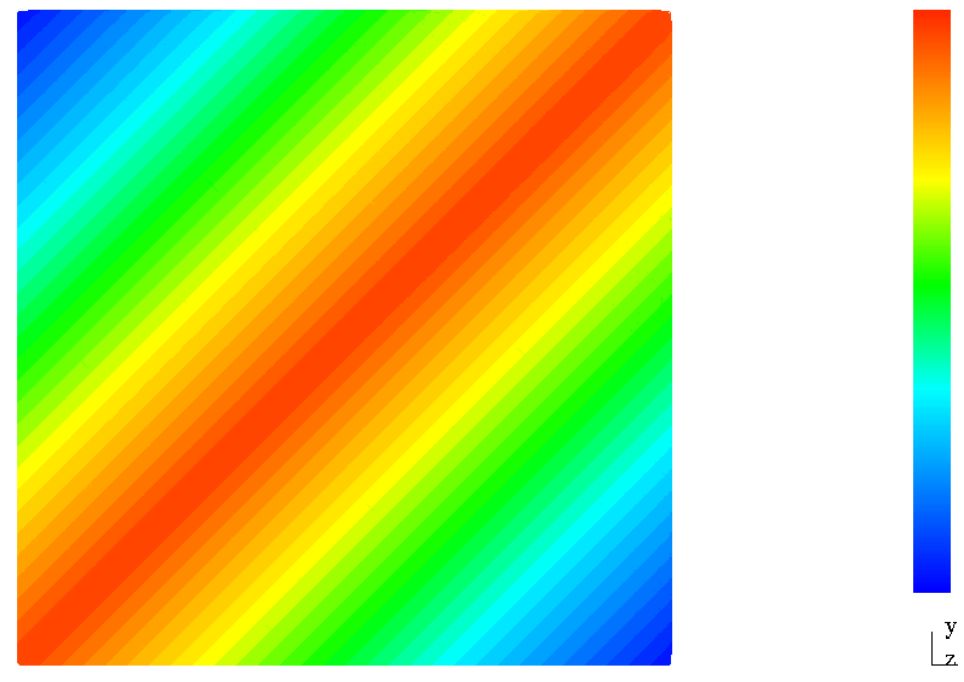

Figure 26: Diffusion parameter $\epsilon=10^{-7}$ : velocity $u$.
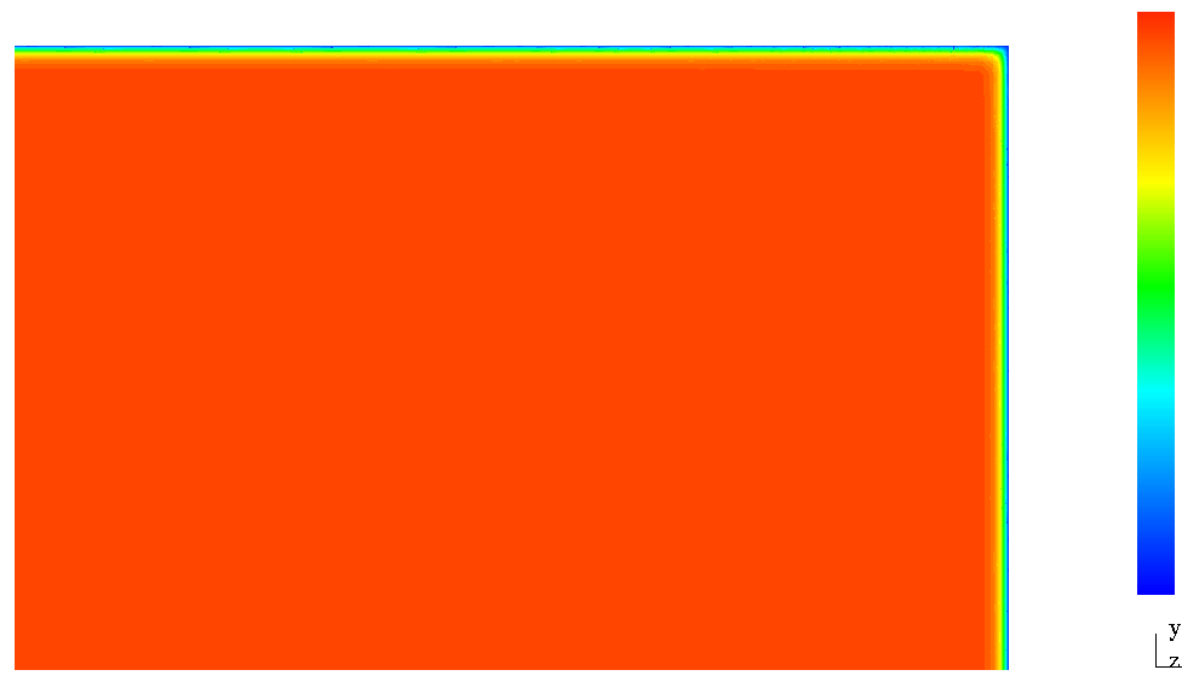

Figure 27: Diffusion parameter $\epsilon=10^{-7}$ : velocity $u, 10^{5}$ zoom on the north-east corner. 

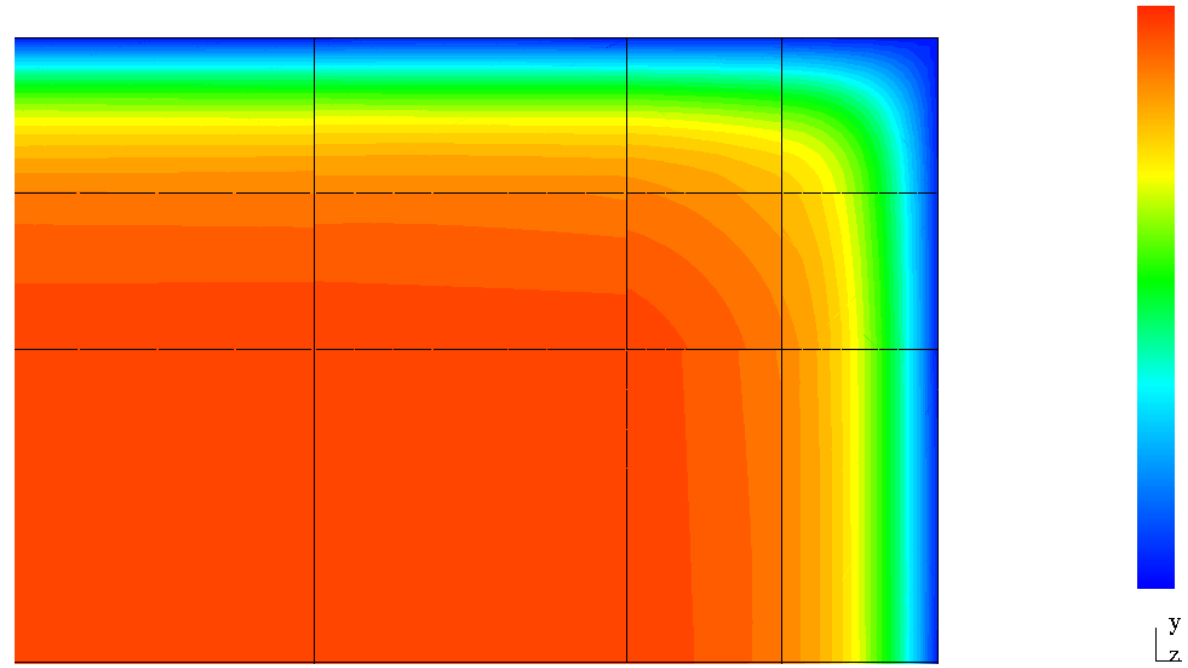

Figure 28: Diffusion paramter $\epsilon=10^{-7}$ : velocity $u, 10^{6}$ zoom on the north-east corner with the mesh.
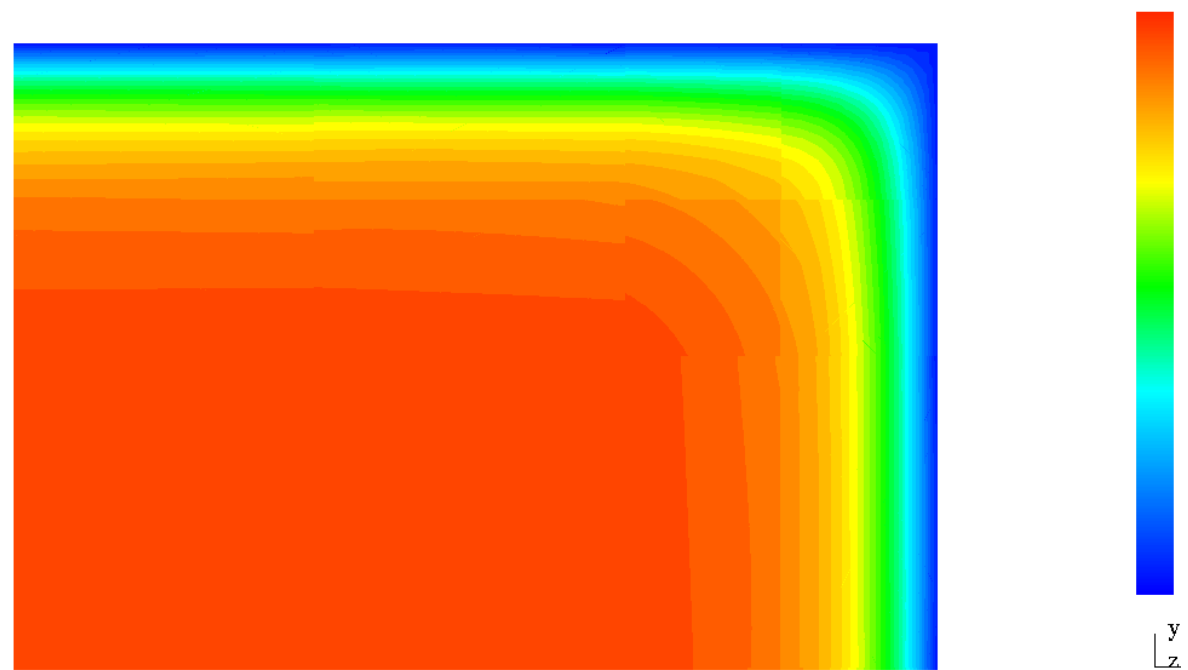

Figure 29: Diffusion parameter $\epsilon=10^{-7}$ : velocity $u, 10^{6}$ zoom on the north-east corner without the mesh. 
proving uniform stability with respect to the mesh. The "natural" DPG energy norm is mesh-dependent, as it is for all DG methods and one of the first tasks in analyzing those methods is to establish a lower bound for the mesh-dependent norm in terms of a global, mesh-independent norm.

We have established such a result for the $1 \mathrm{D}$ convection-dominated diffusion problem using the continuity of the error representation function. An alternative argument based on a concept of globally optimal test functions is presented in Appendix C.

With a proper choice of a weight for the norm in the test space, we have also managed to construct a "robust" version of the method with stability properties uniform in the diffusion parameter $\epsilon$. Construction of "robust" discretization schemes for singularly perturbed problems in general, and for convection-dominated problems in particular, has been a subject of an intensive research for decades, see [25] for a recent extensive review of related work. Even the definition of what we mean by the "robustness" is not easy. Intuitively, given a mesh, we expect the numerical solution to behave uniformly in terms of $\epsilon$ in an "eye-ball" norm if the exact solution behaves so. For the 1D example studied in this paper, the $L^{2}$-norm of both $u$ and $\sigma=\epsilon u^{\prime}$ can be bounded uniformly in $\epsilon$, and we expect the same for the $L^{2}$-norm of the corresponding FE errors. As the first step towards a mathematically rigorous proof of robustness, we have proved that, with a proper choice of weight $\alpha$, the $L^{2}$ error of $u, \sigma$ is bounded by the energy error of the group variable including $u, \sigma$ and the DPG fluxes $\hat{u}, \hat{\sigma}$. The method delivers the best approximation in the energy norm. In $1 \mathrm{D}$, fluxes are just numbers and the best approximation of fluxes is thus always equal zero. Consequently, the $L^{2}$ error of $u, \sigma$ is bounded uniformly by the best approximation error of the same $u, \sigma$ but in a different (the energy) norm. This does not automatically imply the robustness. The $L^{2}$-error in $u$ for the classical Bubnov-Galerkin method is bounded uniformly by the best approximation error of $u$ measured in the $H^{1}$ norm, but the method is not robust as the best approximation error measured in $H^{1}$ norm blows up with $\epsilon$ (see [25] for a detailed discussion). In order to claim the robustness for the DPG method, we will need to establish such a uniform bound for the best approximation error measured in the energy norm.

The choice of the test space norm also turned out to be a crucial tool in the fight against round off error effects. With the rescaled norms, we have been able to extend the current limit of the diffusion parameter $\epsilon$ for which 2D problems can be solved with high-order elements on a double precision platform from to $\epsilon=10^{-7}$. This "pushes the envelope" from DG results obtained by Houston et al. with predefined geometrically graded meshes [17] (Example 3 on page 2159, $\epsilon=10^{-5}$ ) and more recent results of Zhu and Schötzau obtained in a fully adaptive mode [18] (Example 2 on page $28, \epsilon=0.510^{-5}$ ).

Most importantly, our adaptive process is fully automatic and it starts with few elements only. The method does not exhibit any preasymptotic range behavior where stability properties do not hold. In other words, one does not need to start with a mesh that reflects an expertise on the problem.

Convergence analysis in multi-dimensions. A careful examination of the arguments used in $1 \mathrm{D}$ to prove the global continuity of the error representation function reveals that we cannot count on the same result in $2 \mathrm{D}$ or $3 \mathrm{D}$. However, repeating the arguments, we learn that in place of continuity we have an orthogonality result: jump in error representation functions must be orthogonal to the trial space for fluxes. We have for instance,

$$
\int_{e}\left[\phi_{K}\right] \delta \hat{u}_{h p} d s=0
$$

for every function $\delta \hat{u}_{h p}$ from the trial space for edge $e$. Here $\left[\phi_{K}\right]$ denotes the jump of the element error representation functions $\phi_{K}$ across the edge. See also Appendix C for related discussion on the relation between globally and locally optimal test functions.

An explicit computation of the energy norm in multi-dimensions does not seem possible, and we will have to resort to a qualitative analysis only.

Current and future work. Besides the theoretical work, our current effort focuses on applying the DPG method to nonlinear problems - Burgers' and compressible Navier-Stokes equations. We hope to present new exciting results in a forthcoming paper. 
Additional comments added during the review process. The presented paper represents the state of our knowledge at the time of the WONAPDE meeting (January 2010) where this work was presented. Since then we have made a significant progress on both theoretical and numerical sides. Among other results, in [26] we came up with a fundamental concept of the optimal test norm. With the use of the optimal test norm, the corresponding energy norm is close to the original norm in the trial space, i.e. the $L^{2}$-norm for the "field" variables $\boldsymbol{\sigma}, u$. This implies automatically a uniform bound of the $L^{2}$-error by the energy error, similarly to the result established in this paper using the weighted norms. For convection-dominated diffusion however, the optimal test norm involves diffusion coefficient $\epsilon$. Consequently, for large elements used away from the boundary layer, determining optimal test functions becomes as difficult as solving the original problem. The idea is not directly applicable to the "confusion" problems and the weighted test norm remains to be our best choice. In [9], we delivered the first multidimensional analysis, for both Poisson and convection-dominated diffusion problems. The analysis went along different lines than predicted. We managed to prove the mesh independence result but we are still unable to prove the robustness. Finally, the method has been successfully extended to 1D compressible Navier-Stokes equations [27] using the weighted norms discussed here.

\section{Acknowledgements}

Demkowicz was supported in part by the Department of Energy [National Nuclear Security Administration] under Award Number [DE-FC52-08NA28615], and by a research contract with Boeing. Gopalakrishnan was supported in part by the National Science Foundation under grant DMS-0713833. Niemi was supported in part by KAUST. We thank Bob Moser and David Young for encouragement and stimulating discussions on the project.

\section{References}

[1] L. Demkowicz, J. Gopalakrishnan, A class of discontinuous Petrov-Galerkin methods. Part II: Optimal test functions, Numer. Meth. Part. D. E.In print.

[2] J. Oden, L. Demkowicz, Applied Functional Analysis for Science and Engineering, Chapman \& Hall/CRC Press, Boca Raton, 2010, second edition.

[3] I. Babuška, Error-bounds for finite element method, Numer. Math 16.

[4] C. Bottasso, S. Micheletti, R. Sacco, The discontinuous Petrov-Galerkin method for elliptic problems, Comput. Methods Appl. Mech. Engrg. 191 (2002) 3391-3409.

[5] C. Bottasso, S. Micheletti, R. Sacco, A multiscale formulation of the discontinuous Petrov-Galerkin method for advectivediffusive problems, Comput. Methods Appl. Mech. Engrg. 194 (2005) 2819-2838.

[6] P. Causin, R. Sacco, A discontinuous Petrov-Galerkin method with Lagrangian multipliers for second order elliptic problems, SIAM J. Numer. Anal. 43.

[7] P. Causin, R. Sacco, C. Bottasso, Flux-upwind stabilization of the discontinuous Petrov-Galerkin formulation with Lagrange multipliers for advection-diffusion problems, M2AN Math. Model. Numer. Anal. 39 (2005) $1087-1114$.

[8] L. Demkowicz, J. Gopalakrishnan, A class of discontinuous Petrov-Galerkin methods. Part I: The transport equation, Comput. Methods Appl. Mech. Engrg.Accepted, see also ICES Report 2009-12.

[9] L. Demkowicz, J. Gopalakrishnan, Analysis of the DPG method for the Poisson problem, Tech. Rep. 37, ICES, submitteed to SIAM J. Num. Anal. (2010).

[10] B. Cockburn, C.-W. Shu, TVB Runge-Kutta local projection discontinuous Galerkin finite element method for conservation laws II: General framework, Mathematics of Computation 52.

[11] B. Cockburn, C.-W. Shu, The local discontinuous Galerkin method for time-dependent convection-diffusion systems, SIAM J. Num. Anal. 35 (1998) 2440-2463.

[12] T. Hughes, A. Brooks, A multidimensional upwind scheme with no crosswind diffusion, in: Finite Element Methods for Convection Dominated Flows (Papers, Winter Ann. Meeting Amer. Soc. Mech. Engrs., New York, 1979), Vol. 34 of AMD, Amer. Soc. Mech. Engrs. (ASME), New York, 1979, pp. 19-35.

[13] D. Arnold, F. Brezzi, M. Fortin, A stable finite element for the stokes equations, Calcolo 21 (4).

[14] F. Brezzi, A. Russo, Choosing bubbles for advection-diffusion problems, Math. Models Methods Appl. Sci. 4.

[15] F. Brezzi, L. Franca, A. Russo, Further considerations on residual free bubbles for advective-diffusive equations, Comput. Methods Appl. Mech. Engrg. 166 (1998) 25-33.

[16] P. Houston, C. Schwab, , E. Süli, Stabilized $h p$-finite element methods for first-order hyperbolic problems, SIAM J. Numer. Anal. 37 (2000) 1618-1643, (electronic).

[17] P. Houston, C. Schwab, , E. Süli, Discontinuous $h p$-finite element methods for advection-diffusion-reaction problems, SIAM J. Numer. Anal. 39 (2002) 2133-2163. 
[18] I. Zhu, Schötzau, A robust a posteriori error estimate for $h p$-adaptive dg methods for convection-diffusion equations, IMA Journal of Numerical Analysis (2010) 1-35Advance Access published April 28, 2010.

[19] J. Bramble, R. Lazarov, J. Pasciak, A least-squares approach based on a discrete minus one inner product for first order systems, Math. Comp 66.

[20] M. Ainsworth, J. Oden, A Posteriori Error Estimation in Finite Element Analysis, Wiley and Sons, Inc., New York, 2000.

[21] L. Demkowicz, J. Oden, R. Rachowicz, A new finite element method for solving compressible Navier-Stokes equations based on an operator splitting method and $h p$ adaptivity, Comput. Methods Appl. Mech. Engrg. 84 (1990) 275-326.

[22] C. Schwab, M. Suri, The $p$ and $h p$ versions of the finite element method for problems with boundary layers, Math. Comput. 65 (216) (1996) 1403-1429.

[23] J. Melenk, $h p$-Finite Element Methods for Singular Perturbations, Springer, Berlin, 2002.

[24] L. Demkowicz, Computing with $h p$ Finite Elements. I.One- and Two-Dimensional Elliptic and Maxwell Problems, Chapman \& Hall/CRC Press, Taylor and Francis, 2006.

[25] H.-G. Roos, M. Stynes, L. Tobiska, Robust Numerical Methods for Singularly Perturbed Differential Equations, 2nd Edition, Vol. 24 of Springer Series in Computational Mathematics, Springer-Verlag, Berlin, 2008.

[26] J. Zitelli, I. Muga, L. Demkowicz, J. Gopalakrishnan, D. Pardo, V. Calo, A class of discontinuous Petrov-Galerkin methods. Part IV: Wave propagation problems, Tech. Rep. 17, ICES, J. Comp. Phys., in review (2010).

[27] J. Chan, L. Demkowicz, R. Moser, N. Roberts, A class of Discontinuous Petrov-Galerkin methods. Part V: Solution of 1d Burgers and Navier-Stokes equations, Tech. Rep. 25, ICES, submitted to J. Comp. Phys. (2010).

\section{Appendix A. Relation Between 1D Spectral and FE Energy Norms}

In this section, we show that the global energy norm corresponding to norm (2.30), with a proper choice of constants $\beta_{k}$, is bounded below with the corresponding spectral norm of the solution premultiplied with order 1 constant.

The local variational problems for determining optimal test functions look as follows.

$$
\begin{cases}\int_{x_{k-1}}^{x_{k}} \alpha \tau^{\prime} \delta \tau^{\prime}+\tau\left(x_{k}\right) \delta \tau\left(x_{k}\right) & \\ =\frac{1}{\epsilon} \int_{x_{k-1}}^{x_{k}} \sigma_{k} \delta \tau+\int_{x_{k-1}}^{x_{k}} u_{k} \delta \tau^{\prime}-\left.(\hat{u} \delta \tau)\right|_{x_{k-1}} ^{x_{k}} & \forall \delta \tau \\ \int_{x_{k-1}}^{x_{k}} \alpha v^{\prime} \delta v^{\prime}+\beta_{k} v\left(x_{k}\right) \delta v\left(x_{k}\right) & \\ =\int_{x_{k-1}}^{x_{k}} \sigma_{k} v^{\prime}-\left.(\hat{\sigma} \delta v)\right|_{x_{k-1}} ^{x_{k}}-\int_{x_{k-1}}^{x_{k}} u_{k} v^{\prime}+\left.(\hat{u} \delta v)\right|_{x_{k-1}} ^{x_{k}} & \forall \delta v\end{cases}
$$

For each flux unknown $\hat{\sigma}\left(x_{k}\right)$, we have an optimal test function which spans across neighboring elements $\left(x_{k-1}, x_{k}\right)$ and $\left(x_{k}, x_{k+1}\right)$. For the first flux $\hat{\sigma}(0)$, the corresponding test function spans over the first element and, similarly, for the last flux $\hat{\sigma}(1)$, the corresponding test function spans over the last element only. Variational problem (A.1) leads to the following differential equations and boundary conditions for the optimal test functions.

$$
\left\{\begin{aligned}
-\left(\alpha \tau^{\prime}\right)^{\prime} & =\frac{1}{\epsilon} \sigma_{k}-u_{k}^{\prime} \\
\alpha\left(x_{k}\right) \tau^{\prime}\left(x_{k}\right)+\beta_{k} \tau\left(x_{k}\right) & =u_{k}\left(x_{k}\right)-\hat{u}\left(x_{k}\right) \\
-\alpha\left(x_{k-1}\right) \tau^{\prime}\left(x_{k-1}\right) & =-u_{k}\left(x_{k-1}\right)+\hat{u}\left(x_{k-1}\right)
\end{aligned}\right.
$$

and

$$
\left\{\begin{aligned}
-\left(\alpha v^{\prime}\right)^{\prime} & =-\sigma_{k}^{\prime}+u_{k}^{\prime} \\
v^{\prime}\left(x_{k}\right)+\beta_{k} v\left(x_{k}\right) & =\sigma_{k}\left(x_{k}\right)-\hat{\sigma}\left(x_{k}\right)-u_{k}\left(x_{k}\right)+\hat{u}\left(x_{k}\right) \\
-v^{\prime}\left(x_{k-1}\right) & =-\sigma_{k}\left(x_{k-1}\right)+\hat{\sigma}\left(x_{k-1}\right)+u_{k}\left(x_{k-1}\right)-\hat{u}\left(x_{k-1}\right)
\end{aligned}\right.
$$


Solving for the optimal test functions, we obtain the following formula for the energy norm

$$
\begin{gathered}
\|(\boldsymbol{\sigma}, \boldsymbol{u}, \hat{\boldsymbol{\sigma}}, \hat{\boldsymbol{u}})\|_{E}^{2}= \\
\sum_{k=1}^{N}\left[\left\|\frac{1}{\epsilon} \int_{x_{k-1}}^{x} \sigma_{k}(s) d s-u_{k}(x)+\hat{u}\left(x_{k-1}\right)\right\|_{1 / \alpha}^{2}+\left\|-\sigma_{k}(x)+u_{k}(x)+\hat{\sigma}\left(x_{k-1}\right)-\hat{u}\left(x_{k-1}\right)\right\|_{1 / \alpha}^{2}\right] \\
+\sum_{k=1}^{N} \frac{1}{\beta_{k}}\left[\left.\left|\frac{1}{\epsilon} \int_{x_{k-1}}^{x_{k}} \sigma_{k}(s) d s-\hat{u}\right|_{x_{k-1}}^{x_{k}}\right|^{2}+|-\hat{\sigma}|_{x_{k-1}}^{x_{k}}+\left.\hat{u}||_{x_{k-1}}^{x_{k}}\right|^{2}\right]
\end{gathered}
$$

with $\hat{u}(0)=\hat{u}(1)=0$. More precisely,

$$
\begin{aligned}
\boldsymbol{\sigma} & =\left(\sigma_{1}, \ldots, \sigma_{N}\right) \\
\boldsymbol{u} & =\left(u_{1}, \ldots, u_{N}\right) \\
\hat{\boldsymbol{\sigma}} & =\left(\hat{\sigma}(0), \hat{\sigma}\left(x_{1}\right), \ldots, \hat{\sigma}(1)\right) \\
\hat{\boldsymbol{u}} & =\left(\hat{u}\left(x_{1}\right), \ldots, \hat{u}\left(x_{N-1}\right)\right)
\end{aligned}
$$

In order to simplify the notation, we will drop indices in field unknowns $\sigma_{k}(x)=\sigma$ and $u_{k}(x)=u$ and some of the independent and integration variables,

$$
\begin{gathered}
\|(\boldsymbol{\sigma}, \boldsymbol{u}, \hat{\boldsymbol{\sigma}}, \hat{\boldsymbol{u}})\|_{E}^{2}= \\
\sum_{k=1}^{N}\left[\left\|\frac{1}{\epsilon} \int_{x_{k-1}}^{x} \sigma-u+\hat{u}\left(x_{k-1}\right)\right\|_{1 / \alpha}^{2}+\left\|-\sigma+u+\hat{\sigma}\left(x_{k-1}\right)-\hat{u}\left(x_{k-1}\right)\right\|_{1 / \alpha}^{2}\right] \\
+\sum_{k=1}^{N} \frac{1}{\beta_{k}}\left[\left.\left|\frac{1}{\epsilon} \int_{x_{k-1}}^{x_{k}} \sigma-\hat{u}\right|_{x_{k-1}}^{x_{k}}\right|^{2}+|-\hat{\sigma}|_{x_{k-1}}^{x_{k}}+\left.\left.\hat{u}\right|_{x_{k-1}} ^{x_{k}}\right|^{2}\right]
\end{gathered}
$$

The element norms are weighted $L^{2}$-norms with weight $1 / \alpha$ (inverse of the weight used for the norm of the test functions).

We start with a simple estimate,

$$
\begin{aligned}
\left|\frac{1}{\epsilon} \int_{0}^{1} \sigma\right| & =\left|\sum_{k=1}^{N}\left(\frac{1}{\epsilon} \int_{x_{k-1}}^{x_{k}} \sigma-\left.\hat{u}\right|_{x_{k-1}} ^{x_{k}}\right)\right| \\
& \leq \sum_{k=1}^{N} \sqrt{\beta_{k}} \frac{1}{\sqrt{\beta_{k}}}\left|\frac{1}{\epsilon} \int_{x_{k-1}}^{x_{k}} \sigma-\hat{u}\right|_{x_{k-1}}^{x_{k}} \mid \\
& \leq\left(\sum_{k=1}^{N} \beta_{k}\right)^{\frac{1}{2}}\left(\sum_{k=1}^{N} \frac{1}{\beta_{k}}\left|\frac{1}{\epsilon} \int_{x_{k-1}}^{x_{k}} \sigma-\hat{u}\right|_{x_{k-1}}^{x_{k}} \mid\right)^{\frac{1}{2}}
\end{aligned}
$$

The estimate suggests choosing $\beta_{k}=h_{k}$. In the same way,

$$
\begin{aligned}
|\hat{\sigma}(1)-\hat{\sigma}(0)| & =\left|\sum_{k=1}^{N}\left(\left.\hat{\sigma}\right|_{x_{k-1}} ^{x_{k}}-\left.\hat{u}\right|_{x_{k-1}} ^{x_{k}}\right)\right| \\
& \leq \sum_{k=1}^{N} \sqrt{\beta_{k}} \frac{1}{\sqrt{\beta_{k}}}|\hat{\sigma}|_{x_{k-1}}^{x_{k}}-\left.\hat{u}\right|_{x_{k-1}} ^{x_{k}} \mid \\
& \leq\left(\sum_{k=1}^{N} \beta_{k}\right)^{\frac{1}{2}}\left(\sum_{k=1}^{N} \frac{1}{\beta_{k}}|\hat{\sigma}|_{x_{k-1}}^{x_{k}}-\left.\left.\hat{u}\right|_{x_{k-1}} ^{x_{k}}\right|^{2}\right)^{\frac{1}{2}}
\end{aligned}
$$


implies a similar estimate for $|\hat{\sigma}(1)-\hat{\sigma}(0)|$.

Assume now that $x \in\left(x_{k-1}, x_{k}\right), k>1$. We have,

$$
-\sigma(x)+u(x)+\hat{\sigma}(0)=-\sigma(x)+u(x)+\hat{\sigma}\left(x_{k-1}\right)-\hat{u}\left(x_{k-1}\right)+\sum_{l-1}^{k-1}\left[-\left.\hat{\sigma}\right|_{x_{l-1}} ^{x_{l}}+\left.\hat{u}\right|_{x_{l-1}} ^{x_{l}}\right]
$$

which implies

$$
\int_{x_{k-1}}^{x_{k}} \frac{1}{\alpha}|-\sigma+u+\hat{\sigma}(0)|^{2} \leq 2 \int_{x_{k-1}}^{x_{k}} \frac{1}{\alpha}\left|-\sigma+u+\hat{\sigma}\left(x_{k-1}\right)+\hat{u}\left(x_{k-1}\right)\right|^{2}+2 \frac{h_{k}}{\alpha_{k}}\left|\sum_{l-1}^{k-1}\left[-\left.\hat{\sigma}\right|_{x_{l-1}} ^{x_{l}}+\left.\hat{u}\right|_{x_{l-1}} ^{x_{l}}\right]\right|^{2}
$$

where $\alpha_{k}=\min _{x \in\left(x_{k-1}, x_{k}\right)} \alpha(x), k>1$ is assumed to be finite.

We use the discrete Cauchy-Schwarz inequality to estimate the second term on the right-hand side,

$$
\frac{h_{k}}{\alpha_{k}}\left|\sum_{l-1}^{k-1}\left[-\left.\hat{\sigma}\right|_{x_{l-1}} ^{x_{l}}+\left.\hat{u}\right|_{x_{l-1}} ^{x_{l}}\right]\right|^{2} \leq \frac{h_{k}}{\alpha_{k}}\left(\sum_{l=1}^{k-1} \beta_{l}\right)\left(\sum_{l-1}^{k-1} \frac{1}{\beta_{l}}|-\hat{\sigma}|_{x_{l-1}}^{x_{l}}+\left.\left.\hat{u}\right|_{x_{l-1}} ^{x_{l}}\right|^{2}\right)
$$

Taking into account that $\sum_{l=1}^{k-1} \beta_{l}=x_{k-1}$ and summing up the estimate in $k$, we get,

$$
\begin{aligned}
\int_{0}^{1} \frac{1}{\alpha}|-\sigma+u+\hat{\sigma}(0)|^{2} & \leq 2 \sum_{k=1}^{N}\left\|-\sigma+u+\hat{\sigma}\left(x_{k-1}\right)+\hat{u}\left(x_{k-1}\right)\right\|_{1 / \alpha}^{2} \\
& +2 \max _{k>1}\left|\frac{x_{k-1}}{\alpha_{k}}\right|\left(\sum_{k=1}^{N} \frac{1}{\beta_{k}}\left[-\left.\hat{\sigma}\right|_{x_{k-1}} ^{x_{k}}+\left.\hat{u}\right|_{x_{k-1}} ^{x_{k}}\right]\right)
\end{aligned}
$$

Notice that for $\alpha(x)=x$, term $\frac{x_{k-1}}{\alpha_{k}}=1$.

We follow the same strategy to estimate the last term. Starting with identity for $x \in\left(x_{k-1}, x_{k}\right), k>1$,

$$
\frac{1}{\epsilon} \int_{0}^{x} \sigma-u(x)=\frac{1}{\epsilon} \int_{x_{k-1}}^{x} \sigma-u(x)+\hat{u}\left(x_{k-1}\right)+\sum_{l=1}^{k-1}\left[\frac{1}{\epsilon} \int_{x_{l-1}}^{x_{l}} \sigma-\left.\hat{u}\right|_{x_{l-1}} ^{x_{l}}\right]
$$

we obtain,

$$
\begin{aligned}
\int_{0}^{1} \frac{1}{\alpha}\left|\frac{1}{\epsilon} \int_{0}^{x} \sigma-u(x)\right|^{2} & \leq 2 \sum_{k=1}^{N}\left\|\int_{x_{k-1}}^{x} \sigma-u+\hat{u}\left(x_{k-1}\right)\right\|_{1 / \alpha}^{2} \\
& +2 \max _{k>1}\left|\frac{x_{k-1}}{\alpha_{k}}\right|\left(\sum_{k=1}^{N} \frac{1}{\beta_{k}}\left[\frac{1}{\epsilon} \int_{x_{k-1}}^{x_{k}} \sigma-\left.\hat{u}\right|_{x_{k-1}} ^{x_{k}}\right]^{2}\right)
\end{aligned}
$$

We can now formulate now our result.

\section{THEOREM 1}

Consider an arbitrary $(\boldsymbol{\sigma}, \boldsymbol{u}, \hat{\boldsymbol{\sigma}}, \hat{\boldsymbol{u}})$ for which norm (A.4) is finite. Set constants $\beta_{k}=h_{k}:=x_{k}-x_{k-1}$ and assume that the weight $\alpha(x)$ has been selected in such a way that $\min _{x \in\left(x_{k-1}, x_{k}\right)} \alpha(x)=: \alpha_{k}$ is finite, for $k=2, \ldots, N$. Let $\sigma$ and $u$ denote the unions of element functions $\sigma_{k}, u_{k}$. Then the spectral energy norm for $(\sigma, u, \hat{\sigma}(0), \hat{\sigma}(1))$ is bounded by by the FE energy norm of $(\boldsymbol{\sigma}, \boldsymbol{u}, \hat{\boldsymbol{\sigma}}, \hat{\boldsymbol{u}})$,

$$
\left\|\frac{1}{\epsilon} \int_{0}^{x} \sigma-u\right\|_{1 / \alpha}^{2}+\|-\sigma+u+\hat{\sigma}(0)\|_{1 / \alpha}^{2}+\left|\frac{1}{\epsilon} \int_{0}^{1} \sigma(s) d s\right|^{2}+|-\hat{\sigma}(1)+\hat{\sigma}(0)|^{2} \leq C\|(\boldsymbol{\sigma}, \boldsymbol{u}, \hat{\boldsymbol{\sigma}}, \hat{\boldsymbol{u}})\|_{E}^{2}
$$

where $C=\left(1+2 \max \left\{1, \max _{k>1} \frac{x_{k-1}}{\alpha_{k}}\right\}\right)$. For $\alpha(x)=x, C=3$. 


\section{Appendix B. Selection of Weight for 1D Convection-Dominated Problems}

Assume a piecewise constant weight:

$$
\alpha(x)= \begin{cases}w & 0<x<y \\ 1 & y<x<1\end{cases}
$$

We want to determine constant $w$ and coordinate $y$ in such a way that

$$
\left|\int_{0}^{1} e^{-\frac{s}{\epsilon}} g(s) d s\right| \leq C \epsilon\|g\|_{1 / \alpha}
$$

with constant $C$ independent of $\epsilon$. Cauchy-Schwarz inequality implies that it is sufficient to select the weight in such a way that

$$
\left\|e^{-\frac{s}{\epsilon}}\right\|_{\alpha} \leq C \epsilon
$$

Direct integration leads to the condition:

$$
\frac{\epsilon}{2}\left\{\left[1-e^{-\frac{2 y}{\epsilon}}\right] w+\left[e^{-\frac{2 y}{\epsilon}}-e^{-\frac{2}{\epsilon}}\right]\right\} \leq C^{2} \epsilon^{2}
$$

or, assuming $C^{2}=2$,

$$
\left[1-e^{-\frac{2 y}{\epsilon}}\right] w+\left[e^{-\frac{2 y}{\epsilon}}-e^{-\frac{2}{\epsilon}}\right] \leq \epsilon
$$

We can request both terms in the sum above to be less than $\epsilon / 2$. In order to bound the second term, it is sufficient to assume that

$$
e^{-\frac{2 y}{\epsilon}} \leq \frac{\epsilon}{2}
$$

or, equivalently,

$$
y \geq-\frac{\epsilon}{2} \ln \left(\frac{\epsilon}{2}\right)
$$

Recall that the $\log$ function grows very slowly. For instance, for $\epsilon=10^{-7},-\ln (\epsilon / 2) \approx 12$. In order to bound the first term by $\epsilon / 2$ it is sufficient to assume that $w \leq \epsilon / 2$.

\section{Appendix C. Globally and Locally Optimal Test Functions}

Although the concept of optimal test functions is valid for any variational formulations, including those involving standard energy spaces, it is the DPG setting that makes the method practical. More precisely, critical is the use of discontinuous test functions ("broken" Sobolev spaces) which enables the local inversion of the Riesz operator. On the other side, the use of discontinuous test functions, and the resulting fluxes, makes the whole formulation mesh-dependent. The issue of mesh independence is, of course, fundamental for any $h$-convergence result.

In this appendix, we establish mesh independence of the energy norm for the 1D problem discussed in Section 2 using different means. Namely, we introduce the concept of globally optimal test functions and discuss their relation with optimal test functions for the DPG method which, for the sake of this discussion, we will identify now as locally optimal test functions. We shall use the 1D convection-dominated model problem for the discussion, but the conclusions made here apply to any 1D problem.

We begin with the variational formulation behind the one-element version of the DPG method.

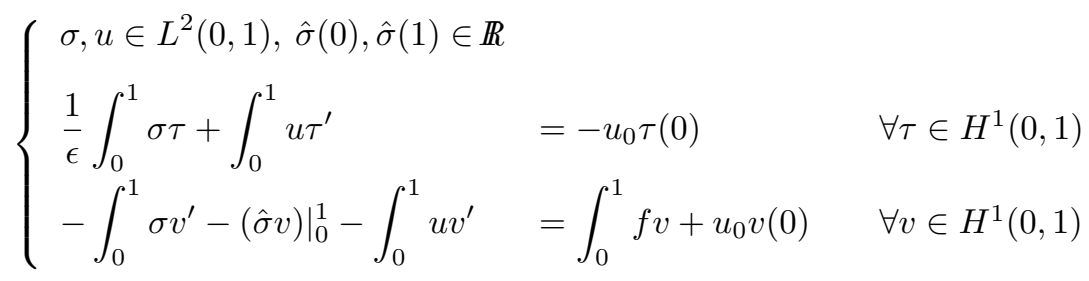


Notice that we do not use the term a spectral method which implies the use of polynomial trial functions defined on interval $(0,1)$. Instead, we assume that field unknowns $\sigma$, $u$ will be approximated with $h p$ trial basis functions corresponding to a particular $h p$ mesh. Contrary to polynomial bases, the $h p$ trial functions are discontinuous (still $L^{2}$-conforming). We can use then the formulation, and the concept of optimal test functions, to introduce the corresponding globally optimal test functions. The optimal test functions are solutions of the corresponding global variational problem,

$$
\begin{cases}\tau, v \in H^{1}(0,1) & \forall \delta \tau \in H^{1}(0,1) \\ \int_{0}^{1} \tau^{\prime} \delta \tau^{\prime}+\tau \delta \tau=\frac{1}{\epsilon} \int_{0}^{1} \sigma \delta \tau+\int_{0}^{1} u \delta \tau^{\prime} & \forall \delta \int_{0}^{1} \sigma \delta v^{\prime}-\left.(\hat{\sigma} \delta v)\right|_{0} ^{1}-\int_{0}^{1} u \delta v^{\prime}\end{cases}
$$

Above, $\sigma, u$ and $\hat{\sigma}(0), \hat{\sigma}(1)$ denote a particular trial function. In practice, the trial functions are scalarvalued, i.e. only one of the terms $\sigma, u$ and $\hat{\sigma}(0), \hat{\sigma}(1)$ is present. Notice that the optimal test function is, in general, vector-valued. The globally optimal test functions are $H^{1}(0,1)$-conforming which makes them globally continuous. As a result of the global continuity of test functions, no interelement fluxes enter the formulation. The corresponding energy norm is mesh-independent. This the good news. The bad news is that the optimal test functions are now global, spanning over the whole mesh. Their determination requires solution of the global problem, and it is as expensive as the solution of the original problem. The resulting stiffness matrix is full and the method is no longer practical.

However, we can ask about the relation between the solutions obtained using the same set of trial functions, and the two sets of globally and locally optimal test functions. The global formulation delivers mesh-independence, the DPG version is practical. What is the relation between the two FE solutions corresponding to the same set of trial functions? Let

$$
0=x_{0}<x_{1}<\ldots x_{k}<x_{k+1}<\ldots x_{N}=1
$$

be a FE mesh covering the domain $(0,1)$. Let $\sigma, u$ and $\hat{\sigma}(0), \hat{\sigma}(1)$ denote a particular trial basis functions corresponding to the mesh, and let $(\tau, v)$ denote the corresponding globally optimal test function. As $\sigma, u$ are smooth within each element (polynomials), variational boundary-value problem (C.2) translates into the following differential equations, boundary and interface conditions for function $\tau$,

$$
\left\{\begin{array}{rlrl}
-\tau^{\prime \prime}+\tau & =\frac{1}{\epsilon} \sigma-u^{\prime} & & \text { in }\left(x_{k-1}, x_{k}\right), k=1, \ldots, N \\
{\left[\left(\tau^{\prime}-u\right)\left(x_{k}\right)\right]} & =0 & & k=1, \ldots, N-1 \\
\tau^{\prime}(1) & =u(1) & & \\
-\tau^{\prime}(0) & =-u(0) &
\end{array}\right.
$$

where $\left[f\left(x_{k}\right)\right]$ denotes the jump of $f$ at $x_{k}$. A similar set of conditions is satisfied by function $v$,

$$
\left\{\begin{array}{rlrl}
-v^{\prime \prime}+v & =\sigma^{\prime}+u^{\prime} & & \text { in }\left(x_{k-1}, x_{k}\right), k=1, \ldots, N \\
{\left[\left(v^{\prime}-\sigma+u\right)\left(x_{k}\right)\right]} & =0 & & k=1, \ldots, N-1 \\
v^{\prime}(1) & =\sigma(1)-\hat{\sigma}(1)-u(1) & \\
-v^{\prime}(0) & =-\sigma(0)=\hat{\sigma}(0)+u(0) &
\end{array}\right.
$$

Let $\delta \tau, \delta v \in \Pi_{k=1}^{N} H^{1}\left(x_{k-1}, x_{k}\right), k=1, \ldots, N$, be now a discontinuous test function. Multiplying $(\text { C.3 })_{1}$ by $\delta \tau$, integrating over each element, and summing up over all elements, we get,

$$
\sum_{k=1}^{N}\left\{-\int_{x_{k-1}}^{x_{k}}\left(\tau^{\prime}-u\right)^{\prime} \delta \tau+\int_{x_{k-1}}^{x_{k}}\left(\tau-\frac{1}{\epsilon} \sigma\right) \delta \tau\right\}=0
$$


Integrating the first term by parts, using the continuity of $v^{\prime}+u$ at element interfaces, and boundary conditions $(\text { C.3 })_{3,4}$, we obtain,

$$
\sum_{k=1}^{N}\left\{\int_{x_{k-1}}^{x_{k}} \tau^{\prime} \delta \tau^{\prime}+\tau \delta \tau\right\}=\sum_{k=1}^{N}\left\{-\int_{x_{k-1}}^{x_{k}} \frac{1}{\epsilon} \sigma \delta \tau+u \delta \tau^{\prime}\right\}+\sum_{k=1}^{N-1}\left(\tau^{\prime}-u\right)\left(x_{k}\right)\left[\delta \tau\left(x_{k}\right)\right]
$$

A similar result is obtained for the $v$-component of the globally optimal test function,

$$
\sum_{k=1}^{N}\left\{\int_{x_{k-1}}^{x_{k}} v^{\prime} \delta v^{\prime}+v \delta v\right\}=\sum_{k=1}^{N}\left\{-\int_{x_{k-1}}^{x_{k}} \sigma v^{\prime}-u v^{\prime}\right\}-\left.(\hat{\sigma} \delta v)\right|_{0} ^{1}+\sum_{k=1}^{N-1}\left(v^{\prime}-\sigma+u\right)\left(x_{k}\right)\left[\delta v\left(x_{k}\right)\right]
$$

As the "broken" test functions $(\delta \tau, \delta v)$ are arbitrary, this proves that the globally optimal test function $(\tau, v)$ is a linear combination of the corresponding locally optimal test function corresponding to the same trial function $\sigma, u, \hat{\sigma}(1), \hat{\sigma}(0)$, and locally optimal test functions corresponding to fluxes $\left(\tau^{\prime}-u\right)\left(x_{k}\right),\left(v^{\prime}-\sigma+u\right)\left(x_{k}\right)$ at the interelement boundaries. This leads to the following result.

\section{THEOREM 2}

Each globally optimal test function can be represented as a linear combination of locally optimal test functions. The globally optimal test functions form a subspace of locally optimal test functions. Consequently, the corresponding FE solutions obtained using either the one element or the DPG setting, are identical.

The result extends to all 1D problems, but the situation in multidimensions is a bit different. Repeating the same reasoning in the $2 \mathrm{D}$ setting, we obtain the variational identities

$$
\begin{gathered}
\sum_{K}\left\{\int_{K} \operatorname{div} \boldsymbol{\tau} \operatorname{div} \boldsymbol{\delta} \boldsymbol{\tau}+\boldsymbol{\tau} \boldsymbol{\delta} \boldsymbol{\tau}\right\}=\sum_{K}\left\{-\int_{K} \frac{1}{\epsilon} \boldsymbol{\sigma} \boldsymbol{\delta} \boldsymbol{\tau}+u \operatorname{div} \boldsymbol{\delta} \boldsymbol{\tau}\right\}+\sum_{e} \int_{e}(\operatorname{div} \boldsymbol{\tau}-u)\left[\delta \tau_{n}\right] \\
\sum_{K}\left\{\int_{K} \nabla v \nabla \delta v+v \delta v\right\}=\sum_{K}\left\{-\int_{K} \boldsymbol{\sigma} \nabla v-\boldsymbol{\beta} u \nabla v\right\}-\int_{\partial K \cap \Gamma} \hat{\sigma}_{n} \delta v+\sum_{e} \int_{e}\left(\frac{\partial v}{\partial n}-\sigma_{n}+\beta_{n} u\right)[\delta v]
\end{gathered}
$$

Here $K$ denote elements, $e$ interelement edges, $\boldsymbol{\beta}$ is the advection vector, and each interelement edge $e$ comes with a predefined normal $\boldsymbol{n}_{e}$ necessary to define fluxes and normal advection vector component $\beta_{n}$. Contrary to $1 \mathrm{D}$, the interelement terms $(\operatorname{div} \boldsymbol{\tau}-u)$ and $\left(\frac{\partial v}{\partial n}-\sigma_{n}+\beta_{n} u\right)$ can only be now approximated with flux trial functions. Consequently, the globally optimal test functions can only be approximated with locally optimal test functions. With too low order for the fluxes, the inf-sup constant for the DPG method is expected to deviate from the inf-sup constant for the one-element formulation.

This intuitively explains the relation of discretization for fluxes with the discretization for the field variables. Increasing order of approximation for fluxes should only improve the global stability. If the approximation for fluxes is insufficient (relative to the approximation for the field variables), we may loose mesh independence. The observation is illustrated with a numerical experiment for a $2 \mathrm{D}$ convection-dominated diffusion problem. We use the data from Section 4 but, for a change, we replace the constant advection with the incompressible velocity field $\boldsymbol{\beta}$ corresponding to potential $\phi$,

$$
\phi=-x^{2}+y^{2}, \quad \beta_{x}=\frac{\partial \phi}{\partial y}, \quad \beta_{y}=-\frac{\partial \phi}{\partial x}
$$

Fig. C.30 presents solutions to the problem after 10 adaptive refinements for different combinations of element and flux orders. If $p$ is the order for the field variables $\left(\sigma_{1}, \sigma_{2}, u\right)$, the order for fluxes is set to (accounting for directionality),

$$
p_{f}=p+\Delta p_{f}
$$

with $\Delta p_{f}=-1,0,1,2$. Diffusion constant is set to a moderate value of $\epsilon=10^{-2}$, and we always start with a mesh of four quadratic elements. Recall that our adaptive strategy is based on $h$-refinements with 

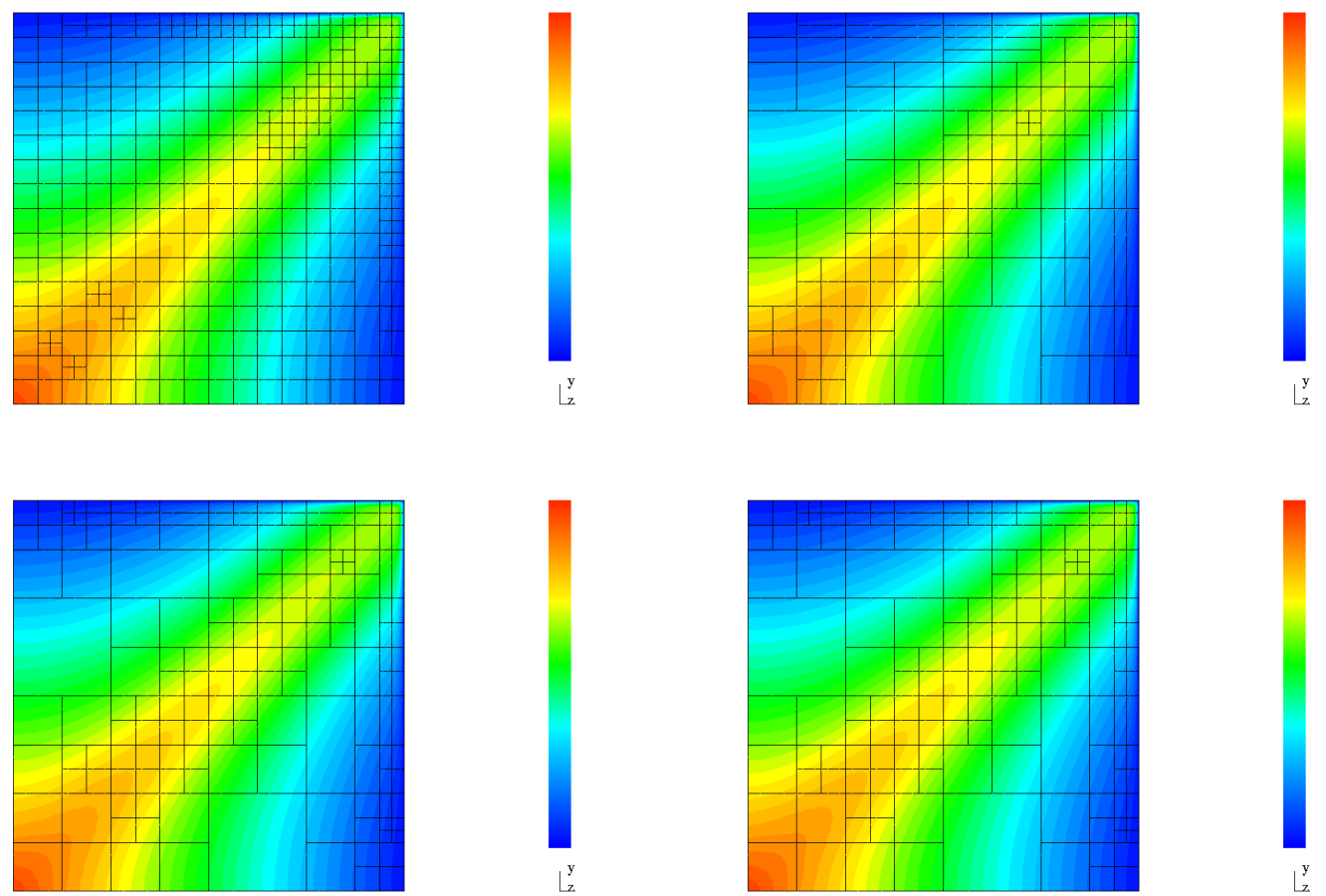

Figure C.30: Convection-dominated diffusion in 2D: solutions after 10 adaptive steps corresponding to $\Delta p_{f}=-1,0,1,2$.

polynomial order being raised only after the element size $h$ drops below $\epsilon$. Increasing order for fluxes makes the method more expensive but, apparently, does not affect the global performance of the method. However, a further decrease of the order for fluxes to $\Delta p_{f}=-2$ (with $p=2$, this means that fluxes are piecewise constant) resulted in a loss of mesh independence. Fig.C.31(a) presents the solution after three adaptive $h$-refinements. Mesh independence is clearly lost and the refinements occur in a wrong place. However, this does not contradict the $p$-convergence of the method for the same mesh as illustrated in Fig.C.31(b) which presents the solution after additional three $p$-refinements. The solution is not converged yet but it is clearly going in the right direction ${ }^{4}$.

\footnotetext{
${ }^{4}$ Further increase in $p$ was limited by the maximum order in our code $p_{\max }=9$ and the use of polynomials of order $p+4$ for the enriched spaces.
} 

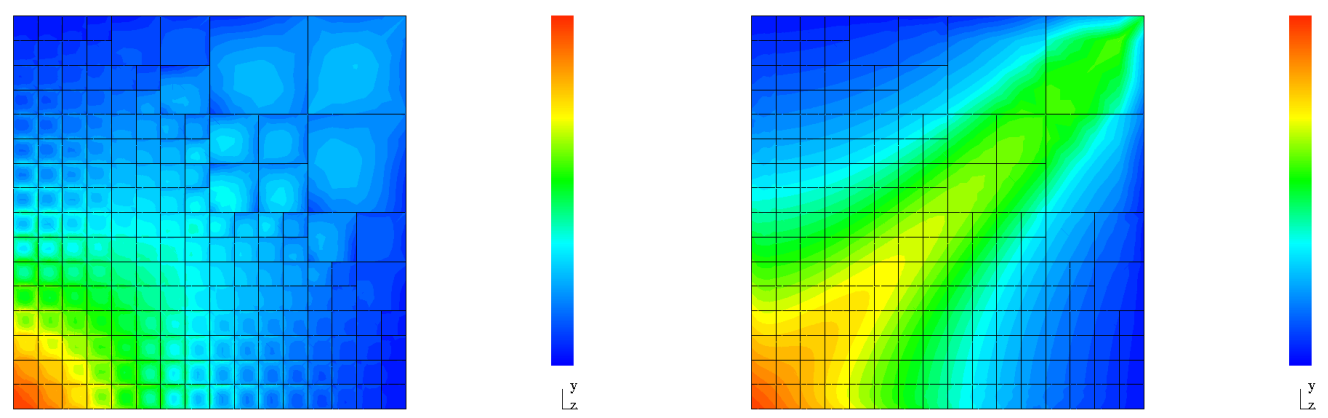

Figure C.31: Convection-dominated diffusion in 2D: (a) solution after 3 adaptive steps corresponding to $\Delta p_{f}=-2$, and (b) after additional three $p$-refinements. 